\title{
Traveling Wavefronts in an Antidiffusion Lattice Nagumo Model*
}

\author{
Maila Brucal-Hallare ${ }^{\dagger}$ and Erik Van Vleck ${ }^{\dagger}$
}

\begin{abstract}
We consider a system of lattice Nagumo equations with cubic nonlinearity, but with a negative discrete diffusion coefficient. We are interested in the existence, uniqueness, stability, and nonexistence of the traveling wavefront solutions of this system, and we shall call this problem the antidiffusion lattice Nagumo problem. By rewriting this system as a spatially periodic system with inhomogeneous but positive periodic diffusion coefficients and periodic nonlinearities, we uncover a rich solution behavior that includes many possible connecting orbits in the antidiffusion case. Second, we observe the presence of bistable and monostable dynamics. In the bistable region, we study the phenomenon of propagation of failure while in the monostable region, we compute the minimum wave speed.
\end{abstract}

Key words. antidiffusion, spatially discrete, reaction-diffusion, traveling waves, bistable, monostable

AMS subject classifications. 39A12, 35K57, 34C 35

DOI. $10.1137 / 100819461$

1. Introduction. In this paper, we consider an antidiffusion model that has been proposed as a simple model for shape memory alloys in which there are twinning microstructures, which arise from the phase transition of the material from the martensite phase to the austenite phase. In studying this problem, lattice differential equations (LDEs) have been proposed because of the importance of the discreteness of the medium. In this paper, we study a particular LDE used to model such phase transitions. In particular, we consider traveling wavefront solutions to an antidiffusion lattice Nagumo equation:

$$
\dot{u}_{n}=d\left(u_{n+1}-2 u_{n}+u_{n-1}\right)-f_{a}\left(u_{n}\right), \quad n \in \mathbb{Z},
$$

where $d<0$, the nonlinearity is the smooth cubic function $f_{a}(u)=u(u-a)(u-1)$, and $a \in \mathbb{R}$. Although much is known about traveling wave solutions to both spatially discrete Nagumo and spatially discrete Fisher systems (with positive diffusion), comparatively little is known about the antidiffusion problem.

Our contribution in this paper is to establish the existence, uniqueness, stability, and nonexistence of traveling wavefront solutions to (1.1). Our approach is to transform the antidiffusion lattice Nagumo problem to a system of spatially discrete reaction-diffusion equations with periodic positive diffusion and periodic nonlinearity terms. We use recent results on traveling waves for bistable and monostable lattice equations in periodic media. In essence, we transform the problem to a system in which the connecting orbits are between spatially

\footnotetext{
* Received by the editors December 28, 2010; accepted for publication (in revised form) by B. Sandstede June 6, 2011; published electronically September 13, 2011. This work was supported in part by NSF grants DMS-0513438 and DMS-0812800.

http://www.siam.org/journals/siads/10-3/81946.html

${ }^{\dagger}$ Department of Mathematics, University of Kansas, Lawrence, KS 66045 (mbrucal@math.ku.edu, evanvleck@ math.ku.edu).
} 
2-periodic solutions of the original negative diffusion problem and uncover a rich structure of traveling wavefront solutions. We have discovered that bistable and monostable dynamics can be observed from the antidiffusion lattice Nagumo problem by varying the values of the parameters $(d, a)$. In the bistable region, we also study the phenomenon of propagation failure while in the monostable region, we study the minimum wave speed. Our results provide a concrete example of traveling wave solutions to a spatially discrete reaction-diffusion system in periodic media.

Both the spatially discrete Nagumo and spatially discrete Fisher equations are examples of LDEs which consist of countably many ordinary differential equations (ODEs) defined over the one-dimensional lattice $\mathbb{Z}$. In contrast with partial differential equations (PDEs), LDEs take into account the spatially discrete structure of the system (see [8] for a review of some results in the theory of LDEs). For example, the spatially discrete Nagumo system has been used as a model for propagation of nerve impulses in myelinated nerve axons [21] while the spatially discrete Fisher system has been used as a model in the spread of a population in patchy environments [19]. Many other examples can be found in neurophysiology, fluid dynamics, and materials science (see [2], [10], [24], [25] and the references therein).

LDEs may also come from a semidiscretization of PDEs by looking at the positive diffusion $d=1 / h^{2}$ as a coupling coefficient and $h$ as the mesh size. The LDEs that we consider in this paper cannot arise from an approximation of PDEs. It comes from a prototypical discrete model of phase transitions that was considered in [25]. This model consists of a chain of particles, each interacting with its nearest and next-to-nearest neighbors in which the longrange interaction between next-to-nearest neighbors is assumed to be harmonic, while the nearest-neighbor interactions are nonlinear and bistable. In the overdamped limit, after a suitable rescaling, a one-dimensional, spatially discrete reaction-diffusion equation with a negative diffusion coefficient and a bistable nonlinearity was obtained. So-called antidiffusion problems in two space dimensions were previously considered in [4], and the rewriting in terms of even and odd lattice sites that we will employ here was described in [5].

The existence, uniqueness, and stability of traveling wavefront solutions to the onedimensional, spatially discrete Nagumo problem has been solved by Zinner [27], [28] for positive diffusion coefficients $d$ that are sufficiently large (see also [16], [17], [19]); on the other hand, Keener [21] showed that only stationary waves exist if $d$ is small. The existence, uniqueness, and stability of traveling wavefront solutions to the one-dimensional, spatially discrete Fisher problem has been solved by Zinner and colleagues [17], [19] and by Chen, Fu, and Guo [6]. Note that, for the Fisher problem, it is essential that there exists a minimum wave speed $c^{*}$ such that for $c \geq c^{*}$, there is a traveling wave solution with wave speed $c$ (see section 5.1.3). In both nonlinearities, as long as we assume a bounded initial condition, we have local existence and uniqueness results for (1.1) whether $d$ is positive or negative.

Solutions to LDEs are sequences $\overrightarrow{\boldsymbol{u}}(t)=\left\{u_{n}(t)\right\}_{n \in \mathbb{Z}}$. Seeking traveling wave solutions requires solving for the wave profile $\phi: \mathbb{R} \rightarrow \mathbb{R}$ and the wave speed $c \in \mathbb{R}$ in the following ansatz:

$$
u_{n}(t)=\phi(n-c t) \quad \text { for every } n \in \mathbb{Z} .
$$

Traveling wavefront solutions, in particular, are traveling wave solutions that have boundary conditions

$$
\phi(-\infty)=\phi_{-}, \quad \phi(+\infty)=\phi_{+},
$$

Copyright (c) by SIAM. Unauthorized reproduction of this article is prohibited. 
so that the traveling wave joins two equilibria $\phi_{-}<\phi_{+}$. Now, the traveling wave ansatz to the spatially discrete equation (1.1) yields a functional differential equation of mixed type (MFDE)

$$
-c \phi^{\prime}(\xi)=d(\phi(\xi+1)-2 \phi(\xi)+\phi(\xi-1))-f_{a}(\phi(\xi)), \quad \text { where } \xi=n-c t,
$$

of which not much is known. Theory for linear MFDEs started to develop in the late 1990s [22], [23], [24]; a general technique for proving existence of traveling waves for certain spatially discrete equations was developed in [23]; and a center-manifold result was developed in [20]. The first stumbling block in solving MFDEs is the fact that the associated initial-value problem is ill-posed [18]. Another quick notable observation is that when $c=0$, the MFDE yields a difference equation with the wave variable $\xi \in \mathbb{Z}$.

In this paper, we will explore the traveling-wavefront problem (existence, uniqueness, stability, and nonexistence) for the antidiffusion lattice Nagumo problem by adapting an approach presented by Chen, Guo, and $\mathrm{Wu}[7]$. They introduced a general framework for the study of traveling wavefront solutions in spatially discrete periodic media using comparison principles, spectrum analysis, and construction of super-/subsolutions. The existence question, in particular, was solved for both the bistable and monostable nonlinearities. If the nonlinearity is bistable, then [7] also proved the uniqueness and stability of traveling waves in periodic media; if the nonlinearity is monostable, then the uniqueness and stability of traveling waves in periodic media was studied in [15]. In this paper, we will show how to restate the antidiffusion lattice Nagumo problem as a periodic heterogeneous media system with periodic positive diffusion coefficients and periodic nonlinearities. The technique used is an appropriate choice of change-of-variables; see section 2 . The resulting system has led us to discover the co-existence of various bistable and monostable dynamical behavior in the antidiffusion lattice Nagumo traveling wavefront problem. This phenomena cannot be observed in the one-dimensional positive diffusion lattice Nagumo system.

It is not obvious that the antidiffusion problem (1.1) can be converted into a 2-periodic system. Section 2 explains the appropriate choice of change-of-variables that is needed for this conversion. Hence, the traveling wavefront problem for the antidiffusion case turns out to be a search for a 2-periodic heteroclinic orbit. To introduce the notion of the period of a traveling wave, we need to look at a slightly different, but equivalent, way of defining a traveling wave solution. A traveling wave solution to a general spatially discrete equation with finite-range interaction

$$
\begin{aligned}
\dot{u}_{n}(t) & =\sum_{k} a_{n, k} u_{n+k}(t)+f_{n}\left(u_{n}(t)\right), \quad n \in \mathbb{Z}, \\
a_{n, k} & =0, \quad|k|>k_{0}, \quad \text { for some integer } k_{0} \geq 1
\end{aligned}
$$

is a solution $\overrightarrow{\boldsymbol{u}}(t)=\left\{u_{n}(t)\right\}_{n \in \mathbb{Z}}$ such that for some constants $c \in \mathbb{R}$ and $T>0$ with $c T \in \mathbb{Z}$

$$
u_{n}(T)=u_{n-c T}(0) \quad \text { for all } n \in \mathbb{Z} .
$$

For such a solution, the number $c$ is called the wave speed and the smallest among the numbers $T$ is called the period. In [7] (see section 3), it was proved that solving for the traveling waves of a spatially discrete $N$-periodic media problem requires solving for $N$ wave profiles.

Copyright $\odot$ by SIAM. Unauthorized reproduction of this article is prohibited. 
This paper is organized as follows. In section 2, we apply a change-of-variables to (1.1), analyze the equilibrium states, and rescale the system so that it is in the framework of [7]. We will see in this section that unlike the positive diffusion problem, there may be more than one possible connecting orbit for the antidiffusion lattice Nagumo problem. In section 3, we present the recent results obtained by Chen, Guo, and $\mathrm{Wu}$ [7] for traveling waves in spatially discrete periodic media. In section 4 , we study the existence, uniqueness, and stability of the traveling wavefront solutions to the antidiffusion lattice Nagumo problem, present the different regions in the plane where there are bistable and monostable dynamics, and look into two special case studies. In section 5, we look at some examples of traveling wavefront problems. With respect to its bistable region, we present some analysis on the phenomena of propagation failure. With respect to its monostable region, we present some preliminary computations for the minimum wave speed of wave solutions that is essential in establishing the existence of solutions. We also present an example showing that there are values for parameters $d, a$ where bistable and monostable connections co-exist. In section 6 , we present some conclusions and avenues for future work.

2. Preliminary computations. In this section, we will show how to transform the antidiffusion problem (1.1) into a system of spatially discrete reaction-diffusion equations with periodic diffusion coefficients and nonlinearities. The first step (see section 2.1) is to relabel the even and odd nodes of (1.1) to obtain a system of two equations that will describe the behavior at the even nodes and the odd nodes, labeling the solution as a two-component vector $(\overrightarrow{\boldsymbol{x}}, \overrightarrow{\boldsymbol{y}})=\left(x_{j}, y_{j}\right)$ for $j \in \mathbb{Z}$; that is, each component of this 2 -vector solution is a sequence of functions of $t$. Then we compute the equilibria of this system (see section 2.2) and observe that unlike the positive diffusion problem, the resulting system will have more than three equilibria (see Lemma 2). These equilibria are the candidates for the boundary conditions $\left(x_{ \pm}, y_{ \pm}\right)$of the traveling wave solution; that is, the traveling wave solution is a heteroclinic orbit connecting $\left(x_{-}, y_{-}\right)$to $\left(x_{+}, y_{+}\right)$, where $x_{ \pm}=\lim _{j \rightarrow \pm \infty} x_{j}$ and $y_{ \pm}=\lim _{j \rightarrow \pm \infty} y_{j}$.

The second step (see section 2.3) is to rescale the system so that $\left(x_{-}, y_{-}\right)$is mapped to $\overrightarrow{\mathbf{0}}=(0,0)$ and $\left(x_{+}, y_{+}\right)$is mapped to $\overrightarrow{\mathbf{1}}=(1,1)$. The result is a system of two equations

$$
\left\{\begin{array}{c}
\dot{v}_{j}=d_{e}\left(w_{j}-2 v_{j}+w_{j-1}\right)-f_{e}\left(v_{j}\right), \\
\dot{w}_{j}=d_{o}\left(v_{j+1}-2 w_{j}+v_{j}\right)-f_{o}\left(w_{j}\right),
\end{array} \quad j \in \mathbb{Z},\right.
$$

with solution $(\overrightarrow{\boldsymbol{v}}, \overrightarrow{\boldsymbol{w}})=\left(v_{j}, w_{j}\right)$ for $j \in \mathbb{Z}$ and diffusion coefficient $\overrightarrow{\boldsymbol{d}}=\left(d_{e}, d_{o}\right)$ and nonlinearity $\vec{f}=\left(f_{e}, f_{o}\right)$, where subscripts $e, o$ stand for the even and odd nodes, respectively. The diffusion coefficients $d_{e}, d_{o}$ are in terms of $d, a$, and we are interested in the case where $d_{e}, d_{o}$, this time, are both positive. An alternative to the change-of-variables outlined here is to apply the transformation $t \mapsto-t$ to the antidiffusion lattice Nagumo problem (1.1) so that the transformed problem has a positive discrete diffusion coefficient but the nonlinearity is no longer of bistable type.

2.1. Even and odd nodes. Let us look at the even and odd lattice nodes of the antidiffusion lattice Nagumo problem

$$
\dot{u}_{n}(t)=d\left(u_{n+1}-2 u_{n}+u_{n-1}\right)-f_{a}\left(u_{n}\right), \quad n \in \mathbb{Z},
$$

Copyright $\odot$ by SIAM. Unauthorized reproduction of this article is prohibited. 
with $d<0$ and cubic nonlinearity $f_{a}(u)=u(u-a)(u-1)$ with $a \in \mathbb{R}$. For completeness, we assume that $a \in \mathbb{R}$ and we mention that, as of this writing, we do not have a physical rationale for considering $a \in \mathbb{R}$. The positive diffusion problem often assumes that $0<a<1$. we get

Writing the even and odd nodes of (2.1) as $\overrightarrow{\boldsymbol{x}}=\left\{x_{j}\right\}_{j \in \mathbb{Z}}$ and $\overrightarrow{\boldsymbol{y}}=\left\{y_{j}\right\}_{j \in \mathbb{Z}}$, respectively,

$$
\left\{\begin{array}{l}
\dot{x}_{j}=d\left(y_{j}-2 x_{j}+y_{j-1}\right)-f_{a}\left(x_{j}\right), \\
\dot{y}_{j}=d\left(x_{j+1}-2 y_{j}+x_{j}\right)-f_{a}\left(y_{j}\right),
\end{array} \quad j \in \mathbb{Z} .\right.
$$

This simple change-of-variables allows us to look at a corresponding two-dimensional system; however, at this point, we cannot perform further analysis because the diffusion-coupling parameter $d$ is negative. What we have done so far is to relabel $\overrightarrow{\boldsymbol{u}}$ as

$$
\begin{aligned}
\overrightarrow{\boldsymbol{u}}(t) & =\left\{\ldots, u_{-2}(t), u_{-1}(t), u_{0}(t), u_{1}(t), u_{2}(t), u_{3}(t), \ldots\right\} \\
& =\left\{\ldots, x_{-1}(t), y_{-1}(t), x_{0}(t), y_{0}(t), x_{1}(t), y_{1}(t), \ldots\right\}
\end{aligned}
$$

At this point, notice that we have only one diffusion parameter $d$; in section 2.3 , after an appropriate change-of-variables, we will have two diffusion parameters that will be periodic and that we will require to both be positive.

2.2. Equilibrium analysis. We need to compute the equilibria that will be used as boundary conditions of the traveling wavefront solutions to (2.2); that is, our goal in this subsection is to compute $\left(x_{ \pm}, y_{ \pm}\right)$defined by

$$
\lim _{j \rightarrow-\infty}\left(x_{j}, y_{j}\right)=\left(x_{-}, y_{-}\right), \quad \lim _{j \rightarrow+\infty}\left(x_{j}, y_{j}\right)=\left(x_{+}, y_{+}\right) .
$$

Note that $\left(x_{ \pm}, y_{ \pm}\right)$are in terms of the parameters $d, a$. The equilibria of (2.2) satisfy

$$
d\left(y_{j}-2 x_{j}+y_{j-1}\right)-f_{a}\left(x_{j}\right)=0=d\left(x_{j+1}-2 y_{j}+x_{j}\right)-f_{a}\left(y_{j}\right), \quad j \in \mathbb{Z},
$$

and hence, as $j \rightarrow \pm \infty$, we obtain

$$
y_{ \pm}=x_{ \pm}+\frac{f_{a}\left(x_{ \pm}\right)}{2 d}, \quad f_{a}\left(x_{ \pm}\right)+f_{a}\left(y_{ \pm}\right)=0 .
$$

In the subsequent discussion, we drop the subscripts \pm . Hence, consider the two equations

$$
y=x+\frac{f_{a}(x)}{2 d}, \quad f_{a}(x)+f_{a}(y)=0,
$$

the solutions of which are the candidates for $\left(x_{ \pm}, y_{ \pm}\right)$. From these two equations, define the ninth-degree polynomial

$$
g(x)=f_{a}(x)+f_{a}\left(x+\frac{f_{a}(x)}{2 d}\right)
$$

Copyright $\odot$ by SIAM. Unauthorized reproduction of this article is prohibited. 


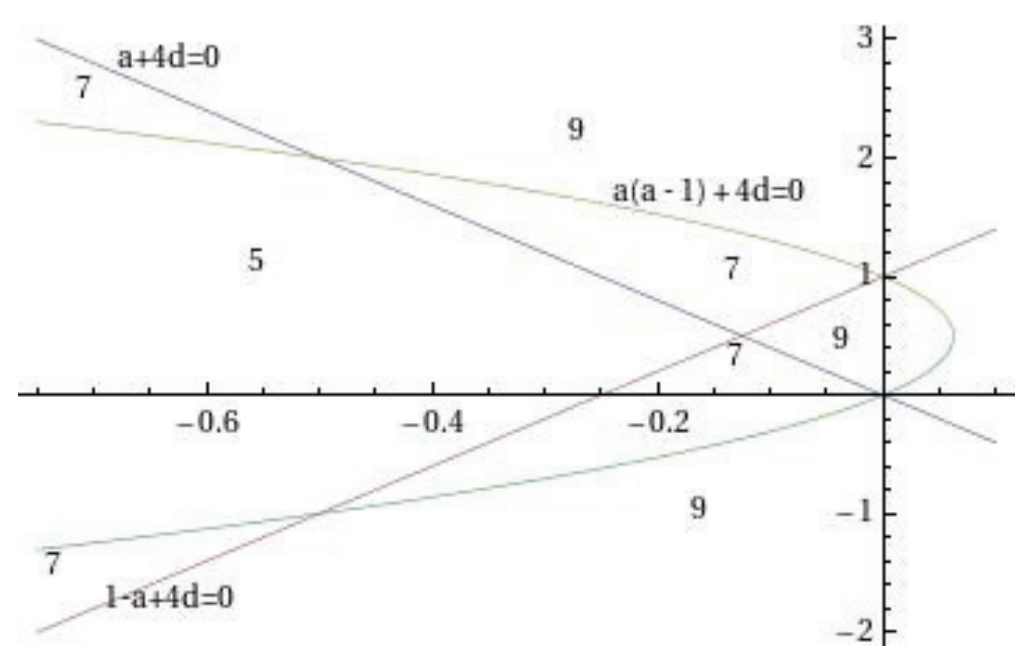

Figure 1. This shows how the number of distinct real zeroes changes in the (d,a)-plane.

The equilibria of (2.2) are zeroes of the polynomial $g$. Computing a closed-form expression of the zeroes of this ninth-degree polynomial in terms of the parameters $d$ and $a$ is not immediate. By applying some simple root analysis, we can characterize the zeroes of $g$, and hence the equilibria of (2.2), completely. These results are contained in Lemma 2.

Lemma 1. Define the curves on the $(d, a)$-plane:

$$
L_{0}=\{a+4 d=0\}, \quad L_{1}=\{1-a+4 d=0\}, \quad L_{a}=\{a(a-1)+4 d=0\} .
$$

Then for $a \neq 0,1$ the following hold:

1. If $(d, a) \in L_{0}$, then 0 is a root of $g$ of multiplicity 3 .

2. If $(d, a) \in L_{1}$, then 1 is a root of $g$ of multiplicity 3 .

3. If $(d, a) \in L_{a}$, then $a$ is a root of $g$ of multiplicity 3 .

Proof. The lemma follows from a direct computation of $g^{\prime}(x), g^{\prime \prime}(x), g^{\prime \prime \prime}(x)$ at $x=0, a, 1$, whose values are tabulated below:

\begin{tabular}{|l|l|l|l|}
\hline$x$ & $2 d g^{\prime}(x)$ & $2 d^{2} g^{\prime \prime}(x)$ & $4 d^{3} g^{\prime \prime \prime}(x)$ \\
\hline 0 & $a(a+4 d)$ & $-(1+a)(a+2 d)(a+4 d)$ & $n_{0}(a, d)$ \\
\hline 1 & $(1-a)(1-a+4 d)$ & $(2-a)(1-a+2 d)(1-a+4 d)$ & $n_{1}(a, d)$ \\
\hline$a$ & $a(a-1)(a(a-1)+4 d)$ & $(2 a-1)(a(a-1)+2 d)(a(a-1)+4 d)$ & $n_{a}(a, d)$ \\
\hline
\end{tabular}

Here $n_{0}, n_{1}, n_{a}$ are polynomials in $d$ and $a$ that are never zero whenever $(d, a) \in L_{0}, L_{1}, L_{a}$, respectively.

The next lemma is our main result on the number of distinct real zeroes of the ninthdegree polynomial $g$ (see Figure 1). This lemma, in particular, claims that there are equilibria of (2.2) that cross 0 and 1 and in some parameter values, there are equilibria in between any two of the three zeroes of the cubic nonlinearity $f_{a}$. The proof uses a simple analysis of the zeroes of $g$ as $(d, a)$ varies on the left-half plane.

Copyright $\odot$ by SIAM. Unauthorized reproduction of this article is prohibited. 
The three curves $L_{0}, L_{1}, L_{a}$ of Lemma 1 divide the left half of the $(d, a)$-plane into seven subregions (see Figure 1), namely

$$
\begin{aligned}
& I=\{1-a+4 d>0, a+4 d>0\}, \\
& I I_{\text {in }}=\{1-a+4 d<0, a+4 d>0, a(a-1)+4 d<0\}, \\
& I I_{\text {out }}=\{1-a+4 d<0, a+4 d>0, a(a-1)+4 d>0\}, \\
& I I I_{\text {in }}=\{1-a+4 d<0, a+4 d<0, a(a-1)+4 d<0\}, \\
& I I I_{\text {out }}=\{1-a+4 d<0, a+4 d<0, a(a-1)+4 d>0\}, \\
& I V_{\text {in }}=\{1-a+4 d>0, a+4 d<0, a(a-1)+4 d<0\}, \\
& I V_{\text {out }}=\{1-a+4 d>0, a+4 d<0, a(a-1)+4 d>0\},
\end{aligned}
$$

where the subscripts in, out serve to indicate that $(d, a)$ is inside or outside the parabola $L_{a}$, respectively. The partitioning gives a picture that is symmetric with respect to the line $a=1 / 2$. The number of zeroes of $g$ will change as the curves $L_{0}, L_{a}, L_{1}$ are crossed. Figure 1 summarizes the results of the following lemma.

Lemma 2. For $a \neq 0,1$ and $d<0, g$ has at least five distinct real zeroes and there exist $\tau_{1}>1, \tau_{2}<0$ such that $g\left(\tau_{i}\right)=0$. Specifically, the following hold:

1. if $(d, a) \in I \cup I I_{\text {out }} \cup I V_{\text {out }}$, then $g$ has nine distinct real zeroes;

2. if $(d, a) \in I I_{\text {in }} \cup I I I_{\text {out }} \cup I V_{\text {in }}$, then $g$ has seven distinct real zeroes; and

3. if $(d, a) \in I I I_{\text {in }}$, then $g$ has five distinct real zeroes.

Proof. From the definition of $g(2.4)$, we can write $g(x)=f_{a}(x) H(x)$, where $H$ is a sixth-degree polynomial with leading coefficient $1 / d^{3}$. We note that

$$
H(0)=\frac{a+4 d}{2 d}, \quad H(1)=\frac{1-a+4 d}{2 d}, \quad H(a)=\frac{a(a-1)+4 d}{2 d}, \quad H( \pm \infty)<0 .
$$

The main components of the proof of this lemma are shown for region $I$. To prove the result for the other regions, one applies similar arguments.

Region I. In this case, we have $0<a<1$ and $a(a-1)+4 d<0$. We claim that $g$ has nine distinct real zeroes; that is, $H$ has six distinct real zeroes, and we will show that two of these are less than 0 , two are greater than 1 , and the other two are located one each in the intervals $(0, a),(a, 1)$.

From (2.5), since both $H(0) H(a)$ and $H(a) H(1)$ are negative, the intermediate value theorem says that there exist $0^{+} \in(0, a), 1^{-} \in(a, 1)$ that are zeroes of $H$. By construction of the polynomial $g$ (see the first equation in 2.3), $0^{+}$gives rise to another zero of $H, 0^{-}:=$ $0^{+}+\frac{f_{a}\left(0^{+}\right)}{2 d}$. Note that $0^{-} \notin\left\{0, a, 1,0^{+}, 1^{-}\right\}$. Moreover, using the second equation in $(2.3)$, it follows that $0^{-}$either is negative or is in the interval $(a, 1)$ because $0<a<1$. Since $0^{+} \in(0, a)$, it follows that $0^{-}<a$ and hence $0^{-}<0$.

Similarly, $1^{-}$defines another zero of $H, 1^{+}:=1^{-}+\frac{f_{a}\left(1^{-}\right)}{2 d} \notin\left\{0, a, 1,0^{+}, 0^{-}, 1^{+}\right\}$. Also, $1^{+}>1$. Now, we have obtained seven zeroes of $g$, ordered as follows: $0^{-}<0<0^{+}<a<$ $1^{-}<1<1^{+}$. Note that each of $0^{ \pm}, 1^{ \pm}$has multiplicity $n=1$; otherwise, if $0^{+}$had multiplicity $n>1$, then $n=2$ and $0^{-}$would have multiplicity 2, too. Since $g^{\prime}(0)<0$ and $g^{\prime}(a)<0$, an even multiplicity for $0^{+}$would give rise to another zero for $H$ in $(0, a)$. However, there is no room for another zero by the fundamental theorem of algebra. A similar argument shows that $1^{+}$has multiplicity 1 . The fundamental theorem of algebra also guarantees that there can be no other zero within the intervals $\left(0^{-}, 0\right)$ and $\left(1,1^{+}\right)$.

Copyright $\odot$ by SIAM. Unauthorized reproduction of this article is prohibited. 
Since $0^{-}$has multiplicity 1 and $g^{\prime}(0)<0<g(-\infty)$, there exists a zero $\tau_{2}<0$ of $H$, distinct from the other four zeroes of $H$. By construction, a sixth zero is $\tau_{1}:=\tau_{2}+\frac{f_{a}\left(\tau_{2}\right)}{2 d} \notin$ $\left\{0, a, 1,0^{ \pm}, 1^{ \pm}, \tau_{2}\right\}$. Thus, we have found all nine zeroes of $g$ whenever $(d, a) \in I$, namely,

$$
\tau_{2}<0^{-}<0<0^{+}<a<1^{-}<1<1^{+}<\tau_{1} .
$$

The zeroes $0^{-}, 0^{+}$coalesce when the boundary curve $L_{0}$ of region $I$ is crossed, while the zeroes $1^{-}, 1^{+}$coalesce when the boundary curve $L_{1}$ is crossed.

The following table is a summary of the subregions and the zeroes of $g$ :

\begin{tabular}{lll}
\hline Region & Range of $a$ & Zeroes of $g$ \\
\hline$I$ & $0<a<1$ & $\tau_{2}<0^{-}<0<0^{+}<a<1^{-}<1<1^{+}<\tau_{1}$ \\
\hline$I I_{\text {in }}$ & $1 / 2<a<1$ & $\tau_{2}<0^{-}<0<0^{+}<a<1<\tau_{1}$ \\
& $a>1$ & $\tau_{2}<0^{-}<0<0^{+}<1<a<\tau_{1}$ \\
\hline$I I_{\text {out }}$ & $a>1$ & $\tau_{2}<0^{-}<0<0^{+}<1<a^{-}<a<a^{+}<\tau_{1}$ \\
\hline$I I I_{\text {in }}$ & $a<0$ & $\tau_{2}<a<0<1<\tau_{1}$ \\
& $0<a<1$ & $\tau_{2}<0<a<1<\tau_{1}$ \\
& $a>1$ & $\tau_{2}<0<1<a<\tau_{1}$ \\
\hline$I I I_{\text {out }}$ & $a>1$ & $\tau_{2}<0<1<a^{-}<a<a^{+}<\tau_{1}$ \\
& $a<0$ & $\tau_{2}<a^{-}<a<a^{+}<0<1<\tau_{1}$ \\
\hline$I V_{\text {in }}$ & $0<a<1$ & $\tau_{2}<0<a<1^{-}<1<1^{+}<\tau_{1}$ \\
& $a<0$ & $\tau_{2}<a<0<1^{-}<1<1^{+}<\tau_{1}$ \\
\hline$I V_{\text {out }}$ & $a<0$ & $\tau_{2}<a^{-}<a<a^{+}<0<1^{-}<1<1^{+}<\tau_{1}$ \\
\hline
\end{tabular}

Observe that we gain or lose two roots every time we cross one of the three bifurcation curves $L_{0}, L_{1}, L_{a}$. There is a saddle-node bifurcation on these three curves because zeroes are either created or annihilated when these curves are crossed. As proved in Lemma 2 and pictured in Figure 1, depending on $a$ (with $d<0$ ), there are three cases when $g$ has nine distinct real zeroes. For example, if $0<a<1$, then $g$ has nine distinct real zeroes whenever $(d, a) \in I: \tau_{2}<0^{-}<0<0^{+}<a<1^{-}<1<1^{+}<\tau_{1}$. The superscripts \pm indicate that the pairs $0^{ \pm}, a^{ \pm}, 1^{ \pm}$are created, respectively, from the saddle-node bifurcation at the curve $L_{0}, L_{a}, L_{1}$.

Unfortunately, we do not have closed-form expressions for the roots $\tau_{i}, 0^{ \pm}, 1^{ \pm}, a^{ \pm}$in terms of $d$ and $a$. Contour plots of $\tau_{1}, \tau_{2}$ for $(d, a) \subseteq(-1 / 4,0) \times(0,1)$ are in Figures 2 and 3, respectively.

Finally, for subsequent reference, define the set

$$
\hat{E}=\left\{(x, y) \in \mathbb{R}^{2}: y=x+\frac{f_{a}(x)}{2 d}, f_{a}(x)+f_{a}(y)=0\right\} .
$$

We shall call a point $(x, y) \in \hat{E}$ an equilibrium of $(2.2)$. The points $\left(x_{ \pm}, y_{ \pm}\right) \in \hat{E}$ are the boundary conditions of the solution $(\overrightarrow{\boldsymbol{x}}, \overrightarrow{\boldsymbol{y}})$. By Lemma 2, the set $\hat{E}$ of equilibria has either five, seven, or nine points, depending on the parameters $(d, a)$ (see Figure 1), where $a \neq 0,1$. In the special case that $\hat{E}$ has nine points, for example when $0<a<1$, the elements of $\hat{E}$ are

$$
\hat{E}=\left\{(0,0),(a, a),(1,1),\left(\tau_{1}, \tau_{2}\right),\left(\tau_{2}, \tau_{1}\right),\left(0^{-}, 0^{+}\right),\left(0^{+}, 0^{-}\right),\left(1^{-}, 1^{+}\right),\left(1^{+}, 1^{-}\right)\right\} .
$$

Copyright $\odot$ by SIAM. Unauthorized reproduction of this article is prohibited. 


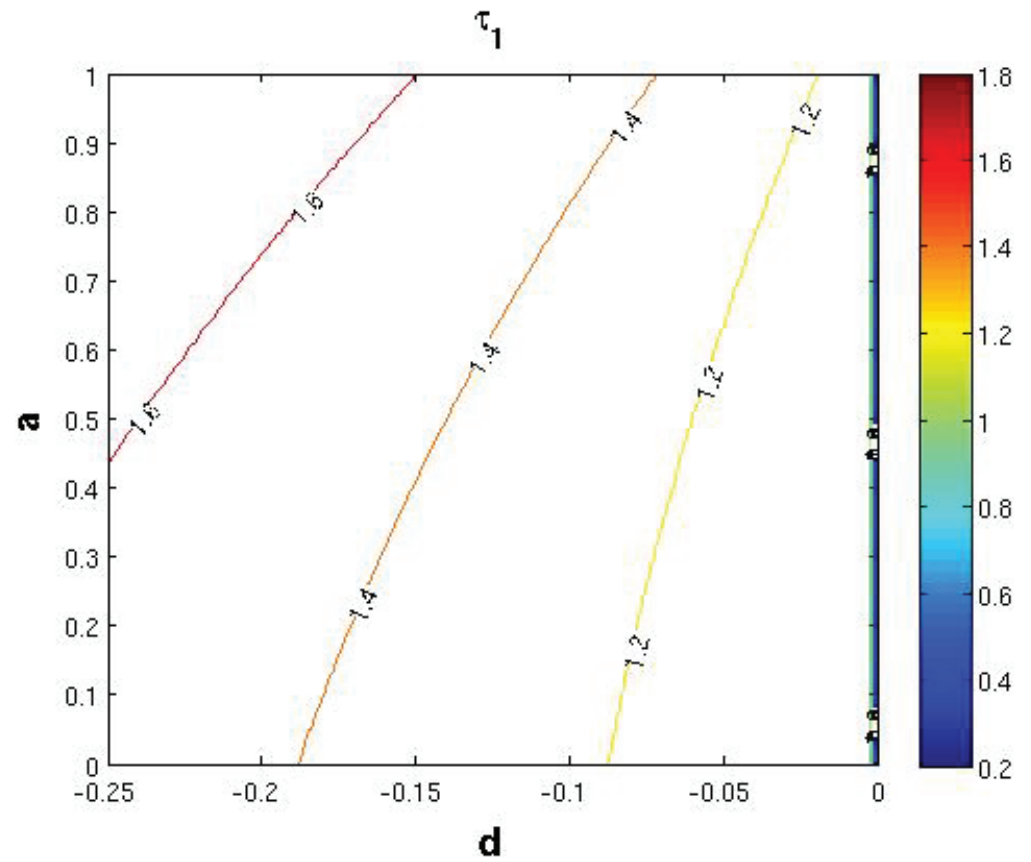

Figure 2. This is the contour plot of $\tau_{1}$ over the region $(d, a) \subseteq(-1 / 4,0) \times(0,1)$. Observe that as $d$ increases to $0, \tau_{1}$ decreases to 1 .

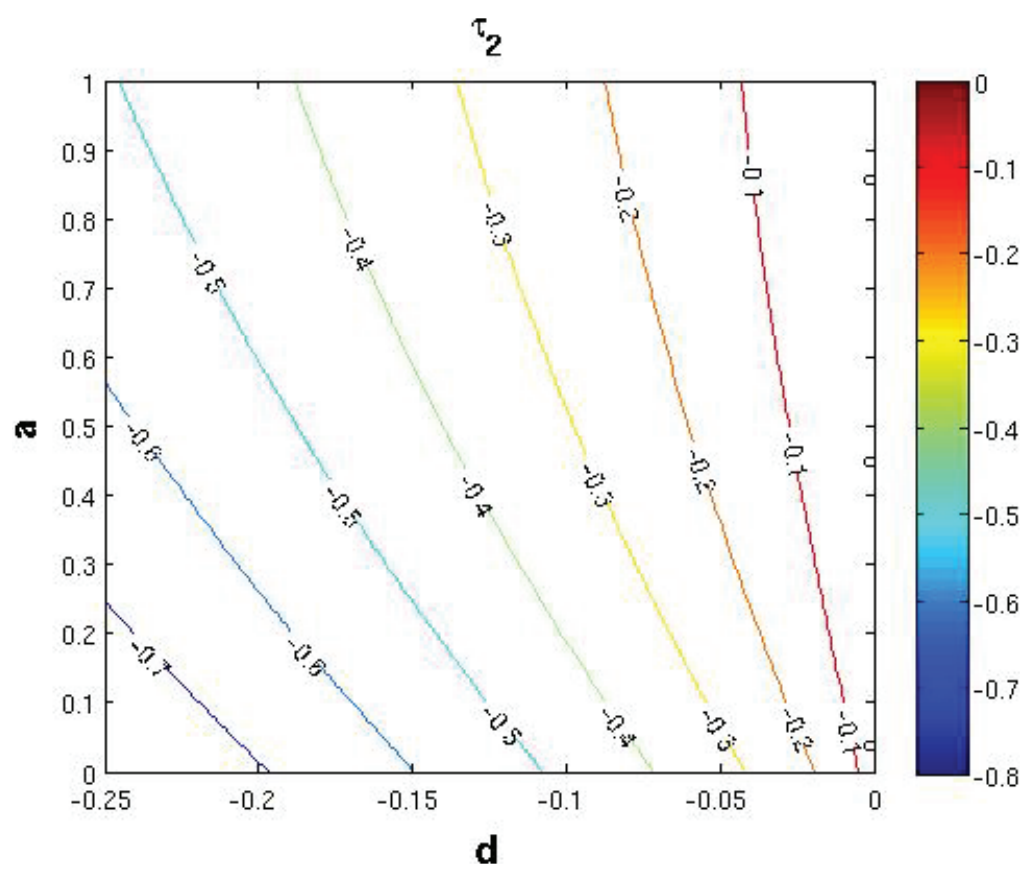

Figure 3. This is the contour plot of $\tau_{2}$ over the region $(d, a) \subseteq(-1 / 4,0) \times(0,1)$. Observe that as $d$ increases to $0, \tau_{2}$ increases to 0. 
2.3. Rescaling the system. We wish to further transform the system (2.2) to a form in which $\left(x_{-}, y_{-}\right) \in \hat{E}$ is mapped to $(0,0)$ and $\left(x_{+}, y_{+}\right) \in \hat{E}$ is mapped to $(1,1)$. Since we are looking for heteroclinic connections, consider two distinct pairs $\left(x_{-}, y_{-}\right)$and $\left(x_{+}, y_{+}\right)$, i.e., $x_{-} \neq x_{+}$and $y_{-} \neq y_{+}$, taken from $\hat{E}$. We employ

$$
v_{j}=\alpha^{-1}\left(x_{j}-x_{-}\right), \quad w_{j}=\beta^{-1}\left(y_{j}-y_{-}\right)
$$

for the even and odd lattice sites, respectively, where

$$
\alpha:=x_{+}-x_{-}, \quad \beta:=y_{+}-y_{-} .
$$

Then upon substituting into (2.2) we obtain

$$
\left\{\begin{array}{l}
\dot{v}_{j}=d_{e}\left(w_{j}-2 v_{j}+w_{j-1}\right)-f_{e}\left(v_{j}\right), \\
\dot{w}_{j}=d_{o}\left(v_{j+1}-2 w_{j}+v_{j}\right)-f_{o}\left(w_{j}\right),
\end{array} \quad j \in \mathbb{Z},\right.
$$

where

$$
d_{e}=\frac{d \beta}{\alpha}, \quad d_{o}=\frac{d \alpha}{\beta}
$$

and the reaction term-nonlinearities are

$$
\begin{aligned}
f_{e}\left(v_{j}\right) & :=\frac{1}{\alpha}\left\{f_{a}\left(\alpha v_{j}+x_{-}\right)+2 d\left[(\alpha-\beta) v_{j}+\left(x_{-}-y_{-}\right)\right]\right\} \\
f_{o}\left(w_{j}\right) & :=\frac{1}{\beta}\left\{f_{a}\left(\beta w_{j}+y_{-}\right)-2 d\left[(\alpha-\beta) w_{j}-\left(x_{-}-y_{-}\right)\right]\right\} .
\end{aligned}
$$

In the above notation, the subscripts $e, o$ of the diffusion parameter $d$ and the nonlinearity $f$ are used to denote even and odd nodes; that is, $d_{e}, d_{o}$ are the diffusion parameters for the even, odd sites, respectively, while $f_{e}, f_{o}$ are the nonlinearity-reaction terms for the even, odd sites, respectively. In fact, we can simplify these two nonlinearities to obtain

$$
f_{e}\left(v_{j}\right)=\alpha^{2} f_{a_{e}}\left(v_{j}\right), \quad f_{o}\left(w_{j}\right)=\beta^{2} f_{a_{o}}\left(v_{j}\right),
$$

where

$$
a_{e}=\frac{-f_{a}^{\prime \prime}\left(x_{-}\right)}{2 \alpha}-1, \quad a_{o}=\frac{-f_{a}^{\prime \prime}\left(y_{-}\right)}{2 \beta}-1,
$$

and we use the notation $f_{a}(u)=u(u-a)(u-1)$. This can be seen by applying the equilibria conditions (2.3) in the following way for the even nonlinearity:

$$
\begin{aligned}
f_{e}\left(v_{j}\right) & =\frac{1}{\alpha}\left\{f_{a}\left(\alpha v_{j}+x_{-}\right)+2 d(\alpha-\beta) v_{j}+2 d\left(x_{-}-y_{-}\right)\right\} \\
& =\frac{1}{\alpha}\left\{f_{a}\left(x_{-}\right)+f_{a}^{\prime}\left(x_{-}\right) \alpha v_{j}+f_{a}^{\prime \prime}\left(x_{-}\right) \frac{\left(\alpha v_{j}\right)^{2}}{2}+\left(\alpha v_{j}\right)^{3}+2 d(\alpha-\beta) v_{j}+2 d\left(x_{-}-y_{-}\right)\right\} \\
& =\frac{1}{\alpha}\left\{f_{a}^{\prime}\left(x_{-}\right) \alpha v_{j}+f_{a}^{\prime \prime}\left(x_{-}\right) \frac{\left(\alpha v_{j}\right)^{2}}{2}+\left(\alpha v_{j}\right)^{3}+2 d(\alpha-\beta) v_{j}\right\} \\
& =\alpha^{2}\left(v_{j}^{3}+\frac{f_{a}^{\prime \prime}\left(x_{-}\right)}{2 \alpha} v_{j}^{2}+\frac{f_{a}^{\prime}\left(x_{-}\right) \alpha+2 d(\alpha-\beta)}{\alpha^{3}} v_{j}\right) \\
& =\alpha^{2} f_{a_{e}}\left(v_{j}\right),
\end{aligned}
$$

Copyright (C) by SIAM. Unauthorized reproduction of this article is prohibited. 
where

$$
a_{e}=\frac{-f_{a}^{\prime \prime}\left(x_{-}\right)}{2 \alpha}-1=\frac{f_{a}^{\prime}\left(x_{-}\right) \alpha+2 d(\alpha-\beta)}{\alpha^{3}},
$$

which follows from the following computation:

$$
\begin{aligned}
& \frac{-f_{a}^{\prime \prime}\left(x_{-}\right)}{2 \alpha}-1-\frac{f_{a}^{\prime}\left(x_{-}\right) \alpha+2 d(\alpha-\beta)}{\alpha^{3}} \\
& =\frac{1}{\alpha^{3}}\left(-f_{a}^{\prime \prime}\left(x_{-}\right) \frac{\alpha^{2}}{2}-\alpha^{3}-f_{a}^{\prime}\left(x_{-}\right) \alpha-2 d(\alpha-\beta)\right) \\
& =\frac{1}{\alpha^{3}}\left(-\left(f_{a}\left(x_{-}\right)+f_{a}^{\prime}\left(x_{-}\right) \alpha+f_{a}^{\prime \prime}\left(x_{-}\right) \frac{\alpha^{2}}{2}+\alpha^{3}\right)+f_{a}\left(x_{-}\right)-2 d(\alpha-\beta)\right) \\
& =-\frac{1}{\alpha^{3}}\left(f_{a}\left(x_{-}+\alpha\right)+f_{a}\left(x_{-}\right)-2 d(\alpha-\beta)\right) \\
& =-\frac{1}{\alpha^{3}}\left(f_{a}\left(x_{+}\right)+f_{a}\left(x_{-}\right)-2 d\left(x_{+}-y_{+}\right)+2 d\left(x_{-}-y_{-}\right)\right) \\
& =0
\end{aligned}
$$

since $2 d\left(x_{ \pm}-y_{ \pm}\right)=-f_{a}\left(x_{ \pm}\right)$. The computations are similar for the odd-site nonlinearity $f_{o}(\cdot)$. We summarize the main result of section 2 .

Lemma 3. By looking at the even and odd nodes, the one-dimensional antidiffusion lattice Nagumo system

$$
\dot{u}_{n}=d\left(u_{n+1}-2 u_{n}+u_{n-1}\right)-f_{a}\left(u_{n}\right), \quad n \in \mathbb{Z},
$$

where $d<0$ and $f_{a}(u)=u(u-a)(u-1), a \in \mathbb{R}$, can be converted into a two-periodic lattice system

$$
\left\{\begin{array}{l}
\dot{v}_{j}=d_{e}\left(w_{j}-2 v_{j}+w_{j-1}\right)-f_{e}\left(v_{j}\right), \\
\dot{w}_{j}=d_{o}\left(v_{j+1}-2 w_{j}+v_{j}\right)-f_{o}\left(w_{j}\right),
\end{array} \quad j \in \mathbb{Z},\right.
$$

where $d_{e}, d_{o}, f_{e}, f_{o}$ are given in (2.10), (2.12), (2.13), (2.8) and

$$
\left(x_{ \pm}, y_{ \pm}\right) \in\left\{y=x+\frac{f_{a}(x)}{2 d}, f_{a}(x)+f_{a}(y)=0\right\} .
$$

In this new system, a traveling wavefront, if it exists, connects $\left(v_{-}, w_{-}\right)=(0,0)$ to $\left(v_{+}, w_{+}\right)=$ $(1,1)$. If $\alpha \beta<0$, then the diffusion coefficients $d_{e}, d_{o}$ are positive.

Thus, if we require $\alpha \beta<0$ in (2.10), we have a system of spatially discrete ODEs with period 2 , with periodic positive diffusion parameters $d_{e}, d_{o}$, and with periodic nonlinearities $f_{e}, f_{o}$. We next summarize the existence, uniqueness, and stability results for traveling wavefronts in spatially discrete periodic media as presented by Chen, Guo, and $\mathrm{Wu}$ [7] and subsequently illustrate the consequences for the antidiffusion lattice Nagumo traveling wavefront problem.

3. Existence, uniqueness, and stability of traveling fronts in discrete periodic media. The classical results on the traveling wavefront problem to spatially continuous [1], [11], [12] or spatially discrete reaction-diffusion equations [21], [27], [28] are under the assumption that the space in which the waves propagate is homogeneous. The simplest nonhomogeneous medium is a periodic medium. The theory presented by Chen, Guo, and $\mathrm{Wu}[7]$ deals with the periodic media case. Unlike the homogeneous medium, the periodic case requires that one solve for $N$ wave profiles, where $N$ is the period of the wave.

Copyright (C) by SIAM. Unauthorized reproduction of this article is prohibited. 
3.1. Assumptions. Here, we state the notation and assumptions for the spatially discrete periodic-media system. Section 3.2 defines the problem while section 3.3 contains the main results [7]. Consider a general system of spatially discrete reaction-diffusion equations for $\vec{U}(t)=\left\{u_{n}(t)\right\}_{n \in \mathbb{Z}}:$

$$
\dot{u}_{n}(t)=\sum_{k} a_{n, k} u_{n+k}(t)+f_{n}\left(u_{n}(t)\right), \quad n \in \mathbb{Z}, \quad t>0,
$$

where the coefficients $a_{n, k}$ are real numbers and have the following assumptions:

A1. Periodic medium. There exists positive integer $N$ such that

$$
a_{n+N, k}=a_{n, k} \quad \text { and } \quad f_{n+N}(\cdot)=f_{n}(\cdot) \text { for all } n, k \in \mathbb{Z} .
$$

A2. Existence of ordered, periodic equilibria. There exist $\overrightarrow{\boldsymbol{\Phi}}^{ \pm}=\left\{\phi_{n}^{ \pm}\right\}_{n \in \mathbb{Z}}$ such that

$$
\sum_{k} a_{n, k} \phi_{n+k}^{ \pm}+f_{n}\left(\phi^{ \pm}\right)=0, \quad \phi_{n+N}^{ \pm}=\phi_{n}^{ \pm}, \quad \phi_{n}^{-}<\phi_{n}^{+}, \quad n \in \mathbb{Z} .
$$

After an appropriate change-of-variables, the equilibria take the form $\overrightarrow{\boldsymbol{\Phi}}^{-}=\overrightarrow{\mathbf{0}}$ and $\overrightarrow{\boldsymbol{\Phi}}^{+}=\overrightarrow{\mathbf{1}}$.

A3. Ellipticity.

$$
a_{n, k}>0 \quad \text { for all } k \neq 0 \quad \text { and } \quad a_{n, 0}=-\sum_{k \neq 0} a_{n, k}<0, \quad n \in \mathbb{Z} .
$$

A4. Nondecoupledness. For every integer pair $i \neq j$, there exist integers $i_{0}, i_{1}, \ldots, i_{m}$ such that $i_{0}=i$ and $i_{m}=j$ with

$$
\prod_{s=0}^{m-1} a_{i_{s}, i_{s+1}-i_{s}}>0 .
$$

A5. Finite-range interaction. There exists a positive integer $k_{0}$ such that

$$
a_{n, k}=0 \quad \text { for } \quad|k|>k_{0} \text { and for all } n \in \mathbb{Z} .
$$

3.2. Statement of the problem. The problem is to find traveling wavefront solutions $(c, \overrightarrow{\boldsymbol{U}})$, where $\overrightarrow{\boldsymbol{U}}(t)=\left\{u_{n}(t)\right\}_{n \in \mathbb{Z}}$ with $t>0$, to the system of equations (3.1) with the stated assumptions that connect two steady states $\overrightarrow{\boldsymbol{\Phi}}^{ \pm}=\left\{\phi_{n}^{ \pm}\right\}_{n \in \mathbb{Z}}$ in the following sense:

$$
\lim _{n \rightarrow \pm \infty}\left[u_{n}(t)-\phi_{n}^{ \pm}\right]=0 \quad \text { for all } t>0 .
$$

We also want to investigate the uniqueness and stability of such solutions. A traveling wave solution $\overrightarrow{\boldsymbol{w}}(\xi)=\left\{w_{n}(\xi)\right\}_{n \in \mathbb{Z}}$ with $\xi \in \mathbb{R}$ is a solution which has the following ansatz:

$$
u_{n}(t)=w_{n}(\xi), \quad \text { where } \xi=n-c t, \quad \text { for all } n \in \mathbb{Z}, t \in \mathbb{R} .
$$

The variable $\xi$ is called the wave variable and the function $\boldsymbol{w}$ is called the wave profile. It can be shown that the wave profile $\overrightarrow{\boldsymbol{w}}$ satisfies

$$
w_{n+N}(\xi)=w_{n}(\xi) \quad \text { for all } n \in \mathbb{Z}, \xi \in \mathbb{R},
$$


and hence there are $N$ functions to solve for, $\left\{w_{1}(\xi), \ldots, w_{N}(\xi)\right\}$, and we will assume that these $N$ wave profiles have a common wave speed $c$. Indeed, for all $x \in \mathbb{R}$ and all $n \in \mathbb{Z}$,

$$
w_{n+N}(\xi)=\left.u_{n+N}(t)\right|_{c t=n+N-x}=u_{n+N}\left(\frac{1}{c}(n+N-x)\right)=u_{n}\left(\frac{1}{c}(n-x)\right)=w_{n}(\xi),
$$

where we have used Theorem 2 (period of the profile).

The next section contains the results of the study of Chen, Guo, and Wu [7]. In particular, they proved the existence of traveling wave fronts in spatially discrete periodic media; however, unlike the spatially continuous homogeneous case, finding a closed form for such solutions is not immediate (see [9] for one technique to construct solutions in the spatially discrete homogeneous media case).

3.3. Results. In this section, we restate the theorems of Chen, Guo, and $\mathrm{Wu}[7]$ on the existence, uniqueness, asymptotics, and stability of a traveling wave solution in spatially discrete periodic media. (See Theorems 2, 3, 4, and 6 in [7].) The first theorem is an existence theorem that asserts that we can find a periodic traveling wavefront solution without requiring any stability condition on the boundary equilibria $\overrightarrow{\mathbf{0}}$ and $\overrightarrow{\mathbf{1}}$. In section 4 , this theorem will be applied to determine the bistable and monostable regions of the antidiffusion lattice Nagumo system.

The following existence theorem is Theorem 6 in [7].

Theorem 1 (see [7]). Assume that $\overrightarrow{\mathbf{0}}, \overrightarrow{\mathbf{1}}$ are steady-states and any other $N$-periodic state $\overrightarrow{\mathbf{\Phi}}=\left\{\phi_{j}\right\}$ with $\phi_{j} \in(0,1)$, if it exists, is unstable. Then the problem (3.1) admits a solution $(c, \overrightarrow{\boldsymbol{w}})$ satisfying

$$
\overrightarrow{\boldsymbol{w}}(-\infty)=\overrightarrow{\mathbf{0}}<\overrightarrow{\boldsymbol{w}}(\xi)<\overrightarrow{\mathbf{1}}=\overrightarrow{\boldsymbol{w}}(+\infty) \quad \text { for all } \xi \in \mathbb{R} .
$$

We have stated the above theorem in a slightly different way; in particular, we included the fact that the other $N$-periodic state $\overrightarrow{\boldsymbol{\Phi}}=\left\{\phi_{j}\right\}$ is such that $\overrightarrow{\mathbf{0}}<\overrightarrow{\mathbf{\Phi}}<\overrightarrow{\mathbf{1}}$, where the ordering should be interpreted componentwise, that is, for all $j, 0<\phi_{j}<1$. Of course, this change is consistent with the proofs and arguments in [7].

The next theorem (Theorems 2, 3, and 4 in [7]) is a result on the uniqueness, asymptotics, and stability of a traveling wave solution when the two steady-states $\overrightarrow{0}, \overrightarrow{\mathbf{1}}$ are both stable.

Theorem 2 (see [7]). Assume that $\overrightarrow{\mathbf{0}}$ and $\overrightarrow{\mathbf{1}}$ are stable steady-states. Suppose that $(c, \overrightarrow{\boldsymbol{U}})$ with $\overrightarrow{\boldsymbol{U}}(t)=\left\{U_{n}(t)\right\}_{n \in \mathbb{Z}}$ is a traveling wave with $c \neq 0$ which connects $\overrightarrow{\mathbf{0}}$ and $\overrightarrow{\mathbf{1}}$.

1. Exponential tail. There exist positive constants $h^{-}, h^{+}$such that

$$
\lim _{n-c t \rightarrow-\infty} \frac{U_{n}(t)}{\psi_{n}^{0} e^{(n-c t) \Lambda^{0}}}=h^{-}, \quad \lim _{n-c t \rightarrow+\infty} \frac{1-U_{n}(t)}{\psi_{n}^{1} e^{(n-c t) \Lambda^{1}}}=h^{+},
$$

where $\left(\left\{\psi_{n}^{0}\right\}_{n \in \mathbb{Z}}, \Lambda^{0}\right)$ and $\left(\left\{\psi_{n}^{1}\right\}_{n \in \mathbb{Z}}, \Lambda^{1}\right)$ with $\Lambda^{1}<0<\Lambda^{0}$ are the eigenvector-eigenvalue pairs of a corresponding eigenvalue problem.

2. Uniqueness. If $(\tilde{c}, \tilde{\boldsymbol{U}})$ is another traveling wave solution, then $c=\tilde{c}$ and there exists $\tau>0$ such that $\tilde{\boldsymbol{U}}(t)=\overrightarrow{\boldsymbol{U}}(t+\tau)$ for all $t \in \mathbb{R}$.

3. Monotonicity in $t$. For all $n \in \mathbb{Z}$ the following hold:

(a) if $c>0$, then $\frac{d}{d t} U_{n}<0$; and

(b) if $c<0$, then $\frac{d}{d t} U_{n}>0$.

Copyright (c) by SIAM. Unauthorized reproduction of this article is prohibited. 
4. Period of the profile. The period of $\overrightarrow{\boldsymbol{U}}$ is $\frac{N}{|c|}$, that is,

$$
U_{n}\left(t+\frac{N}{c}\right)=U_{n-N}(t) \quad \text { for all } t \in \mathbb{R}, n \in \mathbb{Z}
$$

5. Exponential stability. $\overrightarrow{\boldsymbol{U}}$ is globally exponentially stable; that is, if the left tail (near $n=-\infty)$ of an initial datum $\overrightarrow{\boldsymbol{u}}(0)$ is in the basin of attraction of $\overrightarrow{\mathbf{0}}$ and the right tail $($ near $n=+\infty)$ is in the basin of attraction of $\overrightarrow{\mathbf{1}}$, then there are constants $K, \tau^{*}$ such that

$$
\left\|\overrightarrow{\boldsymbol{u}}(t)-\overrightarrow{\boldsymbol{U}}\left(t+\tau^{*}\right)\right\|_{\infty} \leq K e^{-\nu t}, \quad t \geq 0,
$$

where $\nu$ is a constant depending only on $\left\{a_{n, k}\right\}_{(n, k) \in \mathbb{Z} \times \mathbb{Z}}$ and $\left\{f_{n}\right\}_{n \in \mathbb{Z}}$.

4. Antidiffusion and traveling waves. In this section, we first show how to view the antidiffusion lattice Nagumo problem in the framework set forth by Chen, Guo, and Wu [7]. Then we will apply the existence theorem as stated in the previous section in order to find conditions under which we have bistable or monostable dynamics.

4.1. Periodic media. In section 2.3, we have seen that computing for the traveling wave solutions $(c, \overrightarrow{\boldsymbol{u}}(t))$ of the antidiffusion lattice Nagumo problem

$$
\left\{\begin{aligned}
\dot{u}_{n} & =d\left(u_{n+1}-2 u_{n}+u_{n-1}\right)-f_{a}\left(u_{n}\right), \quad n \in \mathbb{Z}, \\
f_{a}(u) & =u(u-a)(u-1), \\
d<0 & \text { and } a \in \mathbb{R}
\end{aligned}\right.
$$

can be converted into a search for 2-periodic solutions $(\overrightarrow{\boldsymbol{v}}(t), \overrightarrow{\boldsymbol{w}}(t))$ of the 2-periodic system

$$
\left\{\begin{array}{c}
\dot{v}_{j}=d_{e}\left(w_{j}-2 v_{j}+w_{j-1}\right)-f_{e}\left(v_{j}\right), \\
\dot{w}_{j}=d_{o}\left(v_{j+1}-2 w_{j}+v_{j}\right)-f_{o}\left(w_{j}\right),
\end{array} \quad j \in \mathbb{Z},\right.
$$

where the diffusion parameters are

$$
d_{e}=\frac{d \beta}{\alpha}, \quad d_{o}=\frac{d \alpha}{\beta}
$$

and the reaction term-nonlinearities are

$$
f_{e}(v)=\alpha^{2} f_{a_{e}}(v), \quad f_{o}(w)=\beta^{2} f_{a_{o}}(w)
$$

with

$$
a_{e}=\frac{-f_{a}^{\prime \prime}\left(x_{-}\right)}{2 \alpha}-1, \quad a_{o}=\frac{-f_{a}^{\prime \prime}\left(y_{-}\right)}{2 \beta}-1,
$$

and $\alpha=x_{+}-x_{-}, \beta=y_{+}-y_{-}$with equilibria $\left(x_{ \pm}, y_{ \pm}\right) \in \hat{E}$. Note that we have already rescaled the system so that $\left(v_{-}, w_{-}\right)=(0,0)$ and $\left(v_{+}, w_{+}\right)=(1,1)$, as required in assumption A2 (see 
section 3.1). We are now in a position to use the results obtained by [7] as presented in section 3. From equations (3.1), we set

$$
\begin{array}{r}
\left\{\begin{array}{c}
a_{n,-1}=d_{e}, \\
a_{n, 0}=-2 d_{e}, \quad n=2 k, \\
a_{n, 1}=d_{e},
\end{array}\right. \\
\left\{\begin{array}{c}
a_{n,-1}=d_{o}, \\
a_{n, 0}=-2 d_{o}, \quad n=2 k+1, \\
a_{n, 1}=d_{o},
\end{array}\right. \\
a_{n, k}=0 \text { for }|k|>1 \text { and for all } n \in \mathbb{Z},
\end{array}
$$

where the diffusion parameters are $d_{e}, d_{o}$ as in (4.3); the nonlinearities are

$$
\begin{cases}f_{n}\left(u_{n}(t)\right)=f_{e}\left(u_{n}(t)\right), & n=2 k \\ f_{n}\left(u_{n}(t)\right)=f_{o}\left(u_{n}(t)\right), & n=2 k+1\end{cases}
$$

with $f_{e}, f_{o}$ as in (4.4); and $a_{e}, a_{o}$ as in (4.5). Thus, we have now fulfilled assumptions A1 and A5 (see section 3.1). Assumption A2 is satisfied with $\phi_{n}^{-}=0, \phi_{n}^{+}=1$ for $n \in \mathbb{Z}$ - this is the rationale for the rescaling process that was performed in section 2.3. In order to satisfy the ellipticity (assumption A3), since we are solving the antidiffusion problem (that is, $d<0$ ), we should require that $\alpha \beta<0$, so that $d_{e}, d_{o}$ are both positive. Finally, assumption A4 is clearly satisfied.

4.2. Existence of solutions. The existence theorem (Theorem 1) in section 3 does not require that the boundary conditions $\overrightarrow{\mathbf{0}}$ and $\overrightarrow{\mathbf{1}}$ are stable or unstable; however, this theorem requires that, in case there are other 2-periodic equilibria, such points must be unstable. In this section, we first study the existence of 2-periodic equilibria, other than $\overrightarrow{\mathbf{0}}$ and $\overrightarrow{\mathbf{1}}$, of the system (4.2) and then perform a linear stability analysis on these points.

4.2.1. Existence of intermediate points. From section 2.3, recall that we have rescaled the system $(2.2)$ so that $\left(v_{-}, w_{-}\right)=(0,0) \equiv \overrightarrow{\mathbf{0}}$ and $\left(v_{+}, w_{+}\right)=(1,1) \equiv \overrightarrow{\mathbf{1}}$ using the change-ofvariables

$$
v:=\frac{x-x_{-}}{\alpha}, \quad w:=\frac{y-y_{-}}{\beta},
$$

where $\left(x_{-}, y_{-}\right) \in \hat{E}$. The set $\hat{E}$ is the set of equilibria of (2.2). Define the set

$$
E=\left\{(v, w): v=\frac{x-x_{-}}{\alpha}, w=\frac{y-y_{-}}{\beta},(x, y) \in \hat{E}\right\} .
$$

We shall call a point $(v, w) \in E$ an equilibrium of (4.2). We are interested in those points $(v, w) \in E$ where $0<v<1,0<w<1$. By ellipticity, we have $\alpha \beta<0$, so that from (4.6),

$$
(v, w) \in E: \quad 0<v<1, \quad 0<w<1
$$

is equivalent to

$$
(x, y) \in E: x_{-}<x<x_{+}, y_{+}<y<y_{-}, \quad \text { or } \quad x_{-}>x>x_{+}, y_{+}>y>y_{-} .
$$

Copyright $\odot$ by SIAM. Unauthorized reproduction of this article is prohibited. 
If there exists a point $(x, y) \in \hat{E}$ such that (4.7) is satisfied, then we shall call the point $(x, y)$ a point intermediate to the boundary conditions $\left(x_{-}, y_{-}\right)$and $\left(x_{+}, y_{+}\right)$in the system (2.2), or simply, an intermediate point. Correspondingly, the point $(v, w) \in E$ will be called a point intermediate to the boundary conditions $\overrightarrow{\mathbf{0}}$ and $\overrightarrow{\mathbf{1}}$ in the system (4.2), or simply, an intermediate point. Because of the change-of-variables (4.6), there is a one-to-one correspondence between the intermediate points of $\hat{E}$ and $E$. There may be equilibria $(x, y) \in \hat{E},(v, w) \in E$ that are not intermediate points.

Example 1 (see section 5.1). Suppose $\left(x_{-}, y_{-}\right)=(0,0)$ and $\left(x_{+}, y_{+}\right)=\left(\tau_{1}, \tau_{2}\right)$. Then $\alpha \beta=$ $\tau_{1} \tau_{2}<0$. In region $I$, the nine zeroes of $g$ are

$$
\tau_{2}<0^{-}<0<0^{+}<a<1^{-}<1<1^{+}<\tau_{1} .
$$

Applying the above criteria, we see that there is exactly one intermediate point $(x, y)=$ $\left(0^{+}, 0^{-}\right)$. In region $I I_{\text {out }}$, the nine zeroes of $g$ are

$$
\tau_{2}<0^{-}<0<0^{+}<1<a^{-}<a<a^{+}<\tau_{1},
$$

so that the only intermediate point is also $(x, y)=\left(0^{+}, 0^{-}\right)$. In region $I V_{\text {out }}$, where $a$ is negative, the nine zeroes of $g$ are

$$
\tau_{2}<a^{-}<a<a^{+}<0<1^{-}<1<1^{+}<\tau_{1},
$$

so that, in this case, there is no intermediate point. For $a>0$, the intermediate point $(x, y)=\left(0^{+}, 0^{-}\right) \in \hat{E}$ in $(v, w)$-coordinates corresponds to $(v, w)=\left(\frac{0^{+}}{\tau_{1}}, \frac{0^{-}}{\tau_{2}}\right) \in E$. This example shows that there is a different behavior for $a<0$ and justifies the study of $a \in \mathbb{R}$, even though the picture in Figure 1 shows that there is symmetry about the line $a=1 / 2$.

Example 2 (see section 5.2). Suppose $\left(x_{-}, y_{-}\right)=\left(\tau_{1}, \tau_{2}\right)$ and $\left(x_{+}, y_{+}\right)=\left(\tau_{2}, \tau_{1}\right)$. Suppose $0<a<1$. In this case, the nine zeroes of $g$, if they exist, are

$$
\tau_{2}<0^{-}<0<0^{+}<a<1^{-}<1<1^{+}<\tau_{1} .
$$

Since the $x_{ \pm}, y_{ \pm}$are the largest and smallest numbers in this list, we see that the other seven pairs are intermediate points. The pairs $\left(0^{ \pm}, 0^{\mp}\right)$ exist provided $a+4 d>0$ while the pairs $\left(1^{ \pm}, 1^{\mp}\right)$ exist provided $1-a+4 d>0$.

Since the set of equilibria $E$ to the system (4.2) has at most nine points and the boundary conditions $\left(x_{-}, y_{-}\right),\left(x_{+}, y_{+}\right) \in \hat{E}$ in a traveling wavefront solution should be distinct, the ellipticity (assumption A3) restricts the number of possible traveling wavefront solutions for any pair of boundary conditions. As we have seen, assumption A3 is equivalent to $\alpha \beta<0$. Hence, for example, the theory does not guarantee that there is a traveling wavefront solution to $(2.2)$ that connects $\left(x_{-}, y_{-}\right)=(0,0)$ to $\left(x_{+}, y_{+}\right)=(1,1)$, since $\alpha=\beta=1$.

In fact, with a fixed $(d, a)$, for each $\left(x_{-}, y_{-}\right) \in \hat{E}$, there are either two, four, six, or eight possible equilibria $\left(x_{+}, y_{+}\right) \in \hat{E}$ such that $\alpha \beta<0$. In each region $(d, a)$ where there are nine points $(x, y) \in \hat{E}$, there are 42 connections from $\left(x_{-}, y_{-}\right)$to $\left(x_{+}, y_{+}\right)$that satisfy $\alpha \beta<0$; that is, there are only 42 pairs (not $9 \cdot 8=72$ ) of boundary conditions from a choice of nine equilibria. In each region region $(d, a)$ where there are seven points $(x, y) \in \hat{E}$, there are 28 connections from $\left(x_{-}, y_{-}\right)$to $\left(x_{+}, y_{+}\right)$that satisfy $\alpha \beta<0$. In the region $(d, a)$ where there 
are five points $(x, y) \in \hat{E}$, there are 14 connections from $\left(x_{-}, y_{-}\right)$to $\left(x_{+}, y_{+}\right)$that satisfy $\alpha \beta<0$. Adding them all up, we will have 252 connections from $\left(x_{-}, y_{-}\right)$to $\left(x_{+}, y_{+}\right)$that satisfy $\alpha \beta<0$ for any given $d, a$. Out of these 252 connections, 116 of them will have an intermediate point.

Next, by Theorem 1, we need to check that every intermediate point $(v, w)$ is unstable. This stability analysis is accomplished in the succeeding discussion.

4.2.2. Stability of equilibria. We want to investigate the (linear) stability of an equilibrium $(v, w) \in E$, intermediate or not. From $(4.6)$, we see that $(v, w)$ is obtained from $(x, y)$ by applying a linear change-of-variables. We observe that the linearization $A(v, w)$ of $(4.2)$ about $(v, w)$ is not symmetric in general because its off-diagonal elements, $2 d_{e}, 2 d_{o}$, may not be equal. The linearization $L(x, y)$ of $(2.2)$ about $(x, y)$ is symmetric. Hence, we study the linear stability of $(v, w)$ by studying the matrix $L(x, y)$, given by

$$
L(x, y)=\left(\begin{array}{cc}
-\left(2 d+f_{a}^{\prime}(x)\right) & 2 d \\
2 d & -\left(2 d+f_{a}^{\prime}(y)\right)
\end{array}\right)
$$

with trace and determinant

$$
T(x, y)=-4 d-\left(f_{a}^{\prime}(x)+f_{a}^{\prime}(y)\right), \quad D(x, y)=2 d\left(f_{a}^{\prime}(x)+f_{a}^{\prime}(y)\right)+f_{a}^{\prime}(x) f_{a}^{\prime}(y) .
$$

Since $L(x, y)$ is symmetric, its two eigenvalues are real so that the point $(x, y)$ is unstable if and only if the larger eigenvalue of $L(x, y)$ is positive. The eigenvalues of $L(x, y)$ are given by $\frac{1}{2}\left(T \pm \sqrt{T^{2}-4 D}\right)$, where $T, D$ are the trace and determinant, respectively.

For $(x, y) \neq(0,0),(a, a),(1,1)$, we use $(2.3)$ to see that $D(x, y) \equiv 0$. Hence, the eigenvalues of $L(x, y)$ are either $T$ or 0 . The eigenvalue $T$ is positive provided $2 d+f_{a}^{\prime}(x)<0$; this comes from

$$
T=-4 d-f_{a}^{\prime}(x)-f_{a}^{\prime}(y)=-4 d-f_{a}^{\prime}(x)+\frac{2 d f_{a}^{\prime}(x)}{2 d+f_{a}^{\prime}(x)}=\frac{-\left[\left(2 d+f_{a}^{\prime}(x)\right)^{2}+4 d^{2}\right]}{2 d+f_{a}^{\prime}(x)} .
$$

For $(x, y)=(0,0),(a, a),(1,1)$, we summarize the trace, determinant, and eigenvalues in the following table:

\begin{tabular}{|c|c|c|c|}
\hline Equilibrium $(x, y)$ & Trace & Determinant & Eigenvalues \\
\hline$(0,0)$ & $-2(a+2 d)$ & $a(a+4 d)$ & $-(a+4 d),-a$ \\
$(1,1)$ & $-2(1-a+2 d)$ & $(1-a)(1-a+4 d)$ & $-(1-a+4 d),-(1-a)$ \\
$(a, a)$ & $-2(a(a-1)+2 d)$ & $a(a-1)(a(a-1)+4 d)$ & $-(a(a-1)+4 d),-a(a-1)$ \\
\hline
\end{tabular}

Lemma 4. Given a fixed $d<0$ and $a \in \mathbb{R}$, suppose $\alpha \beta<0$. The following are equivalent:

1. the equilibrium $(v, w) \in E$ is a 2-periodic unstable solution of (4.2);

2. the equilibrium $(x, y) \in \hat{E}$ is a 2-periodic unstable solution of (2.2);

3. $2 d+f_{a}^{\prime}(x)<0$, where $x \notin\{0, a, 1\}$;

4. $2 d+f_{a}^{\prime}(y)<0$, where $y \notin\{0, a, 1\}$;

5. $f_{a}^{\prime}(x) f_{a}^{\prime}(y)<8 d^{2}$, where $x, y \notin\{0, a, 1\}$.

In particular, if $f_{a}^{\prime}(x) f_{a}^{\prime}(y)<0$, then $(x, y)$ (and hence $\left.(v, w)\right)$ is unstable.

Copyright (C) by SIAM. Unauthorized reproduction of this article is prohibited. 


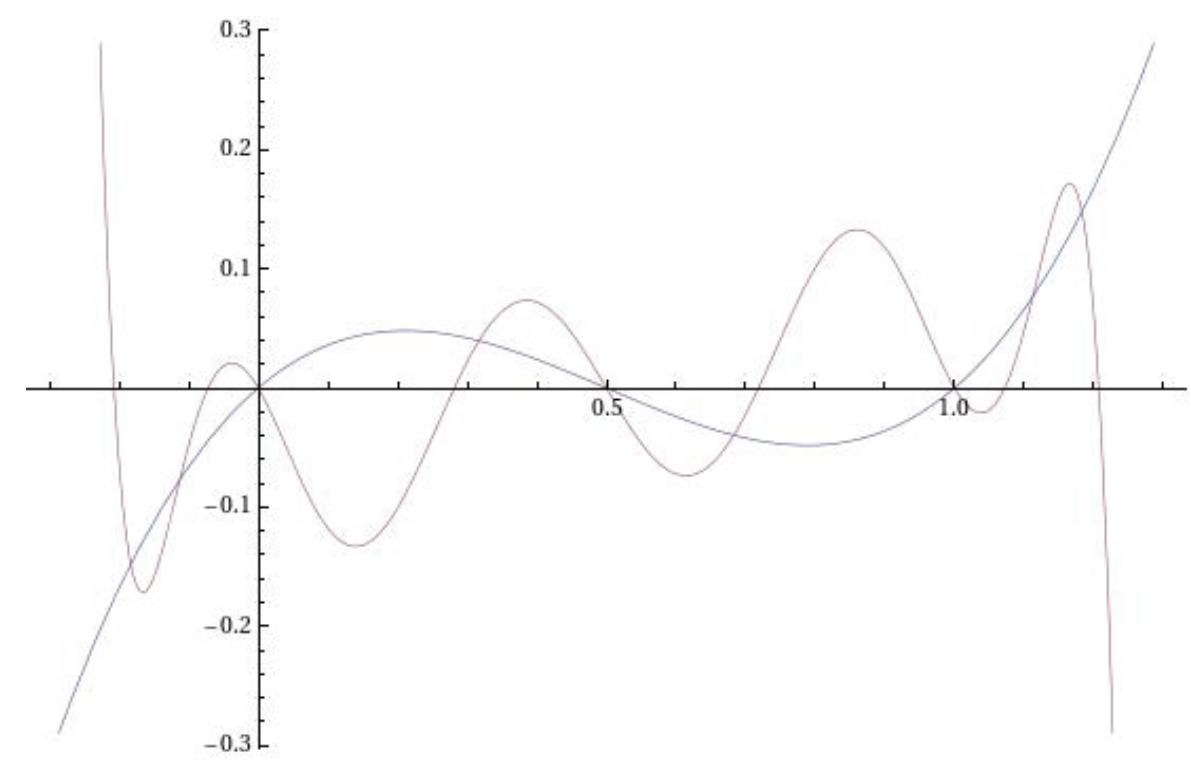

Figure 4. This shows the graphs of $f_{a}$ and $g$ in the Cartesian plane when $a=1 / 2, d=-1 / 16$. In this case, the spinodal interval is $\left(\frac{1}{2}-\frac{\sqrt{3}}{6}, \frac{1}{2}+\frac{\sqrt{3}}{6}\right)$ so that $f_{a}^{\prime}\left(0^{-}\right) f_{a}^{\prime}\left(0^{+}\right)<0$ and $f_{a}^{\prime}\left(1^{-}\right) f_{a}^{\prime}\left(1^{+}\right)<0$.

Testing analytically that the inequalities in this lemma are true is not immediate because we do not have closed-form expressions for $x$ and $y$ as zeroes of the ninth-degree polynomial $g$. However, we can formulate some simple observations that will allow us to check $f_{a}^{\prime}(x) f_{a}^{\prime}(y)<0$ for some pairs $(x, y)$ (see Figure 4 ). Define the spinodal interval of $f_{a}$ :

$$
S=\left(s_{-}, s_{+}\right), \text {where } s_{ \pm}=\frac{1}{3}\left(a+1 \pm \sqrt{a^{2}-a+1}\right) .
$$

Note that for any $a \in \mathbb{R}, a^{2}-a+1>0$. The endpoints of the spinodal interval are the points where the derivative of $f_{a}$ changes signs.

Lemma 5. If $x \in S$ and $y \notin S$ or $y \in S$ and $x \notin S$, then $f_{a}^{\prime}(x) f_{a}^{\prime}(y)<0$ so that $(x, y)$ is unstable.

Example 3. See Figure 4, where the given $(d, a)$-values are in region $I$. The components of the equilibria

$$
(0,0),\left(\tau_{1}, \tau_{2}\right),\left(\tau_{2}, \tau_{1}\right),(1,1)
$$

are all outside $S$ while the components of the point $(a, a)$ are inside $S$. Hence, these five points are stable. The other four equilibria, $\left(0^{ \pm}, 0^{\mp}\right)$ and $\left(1^{ \pm}, 1^{\mp}\right)$, are unstable. From Example 1 and by the previous lemma, we see that $\left(0^{+}, 0^{-}\right)$is the only intermediate unstable equilibrium between the two stable equilibria $\left(x_{-}, y_{-}\right)=(0,0)$ and $\left(x_{+}, y_{+}\right)=\left(\tau_{1}, \tau_{2}\right)$. Similarly, $\left(1^{+}, 1^{-}\right)$ is the only intermediate unstable equilibrium between the two stable equilibria $\left(x_{-}, y_{-}\right)=$ $(1,1)$ and $\left(x_{+}, y_{+}\right)=\left(\tau_{1}, \tau_{2}\right)$.

Note that $2 d+f_{a}^{\prime}(x)=3 x^{2}-2(a+1) x+a+2 d$ and for $d<0$, we have $Q(a, d):=$ $a^{2}-a+1-6 d>0$. Hence if

$$
\frac{a+1-\sqrt{Q(a, d)}}{3}<x<\frac{a+1+\sqrt{Q(a, d)}}{3},
$$

Copyright $\odot$ by SIAM. Unauthorized reproduction of this article is prohibited. 
then $2 d+f_{a}^{\prime}(x)<0$ so that $(x, y)$ is unstable. Otherwise, if

$$
x<\frac{a+1-\sqrt{Q(a, d)}}{3} \quad \text { o } \quad x>\frac{a+1+\sqrt{Q(a, d)}}{3},
$$

then $2 d+f_{a}^{\prime}(x)>0$ so that $(x, y)$ is stable.

Finally, our discussion of stability of equilibria agrees with the stability criteria in [7] (see Theorem 1, page 197), where they have proven that the characteristic equation has at most two real roots.

4.2.3. Bistable and monostable dynamics. In this final subsection, we define what it means for the system (4.2) to exhibit bistable and monostable dynamics and then derive conditions under which (4.2) has such dynamics.

Bistable dynamics corresponds to $\overrightarrow{\mathbf{0}}$ and $\overrightarrow{\mathbf{1}}$ being stable 2-periodic equilibrium solutions to (4.2) and an unstable 2-periodic equilibrium solution $\overrightarrow{\boldsymbol{a}}=(v, w)$ with $0<v<1$ and $0<w<1$ and no other stable 2-periodic equilibrium solutions $(v, w)$ with values in $(0,1) \times(0,1)$.

Monostable dynamics corresponds to one of $\overrightarrow{0}$ or $\overrightarrow{\mathbf{1}}$ being a stable 2-periodic equilibrium solution and the other an unstable 2-periodic equilibrium solution and no other stable 2periodic equilibrium solutions with values in $(0,1) \times(0,1)$.

Theorem 3. Given a fixed $d<0$ and $a \in \mathbb{R}$, let $\left(x_{ \pm}, y_{ \pm}\right) \in \hat{E}$. Suppose $(x, y) \in \hat{E}$ is an intermediate point between $\left(x_{-}, y_{-}\right)$and $\left(x_{+}, y_{+}\right)$:

1. If $f_{a}^{\prime}\left(x_{ \pm}\right) f_{a}^{\prime}\left(y_{ \pm}\right) \geq 8 d^{2}>f_{a}^{\prime}(x) f_{a}^{\prime}(y)$, then both $\left(x_{-}, y_{-}\right)$and $\left(x_{+}, y_{+}\right)$are stable and $(x, y)$ is unstable. If there are no other stable 2-periodic equilibria solutions, then the antidiffusion lattice Nagumo system exhibits bistable dynamics.

2. If $f_{a}^{\prime}\left(x_{-}\right) f_{a}^{\prime}\left(y_{-}\right) \geq 8 d^{2}>f_{a}^{\prime}\left(x_{+}\right) f_{a}^{\prime}\left(y_{+}\right)$, then $\left(x_{-}, y_{-}\right)$is stable while $\left(x_{+}, y_{+}\right)$is unstable; or if $f_{a}^{\prime}\left(x_{+}\right) f_{a}^{\prime}\left(y_{+}\right) \geq 8 d^{2}>f_{a}^{\prime}\left(x_{-}\right) f_{a}^{\prime}\left(y_{-}\right)$, then $\left(x_{+}, y_{+}\right)$is stable while $\left(x_{-}, y_{-}\right)$is unstable. If there are no other stable 2-periodic solutions, then the antidiffusion lattice Nagumo system exhibits monostable dynamics.

\section{Examples.}

\subsection{Case study: $(0,0)$ to $\left(\tau_{1}, \tau_{2}\right)$ connection.}

5.1.1. Existence. In section 2 , we have seen that for any value of the parameter $(d, a)$ for $d<0$, there always exist numbers outside the interval $(0,1)$ that give rise to equilibria to the system (2.2); that is, there exist $\tau_{1}>1$ and $\tau_{2}<0$ with $g\left(\tau_{i}\right)=0$ such that $\left(\tau_{1}, \tau_{2}\right)$ or $\left(\tau_{2}, \tau_{1}\right)$ is an equilibrium of (2.2). We want to know whether there exists a traveling wavefront solution to $(2.2)$ that connects $\left(x_{-}, y_{-}\right)=(0,0)$ and $\left(x_{+}, y_{+}\right)=\left(\tau_{1}, \tau_{2}\right)$. (In the positive diffusion problem, the traveling wavefront solution that is often studied is a solution that connects 0 to 1.$)$

In this case, $\alpha=\tau_{1}>1, \beta=\tau_{2}<0$. We set $v_{j}=\frac{x_{j}}{\tau_{1}}, w_{j}=\frac{y_{j}}{\tau_{2}}$. In system (4.2), the diffusion-coupling parameters are $d_{e}=\frac{d \tau_{2}}{\tau_{1}}, d_{o}=\frac{d \tau_{1}}{\tau_{2}}$, and the reaction-nonlinearity terms are $f_{e}(v)=\tau_{1}^{2} f_{a_{e}}(v), f_{o}(w)=\tau_{2}^{2} f_{a_{o}}(w)$ with detuning parameters $a_{e}=\frac{a+1}{\tau_{1}}-1, a_{o}=\frac{a+1}{\tau_{2}}-1$, respectively. If $a>0$, then the three detuning parameters are related by $a_{o}<0<a<a_{e}$. Figure 5 shows a contour plot of $a_{e}$ for $-0.25<d<0$ and $0<a<1$ where we observe that when $a+4 d>0$, the detuning parameter $a_{e} \in(0,1)$.

Copyright (c) by SIAM. Unauthorized reproduction of this article is prohibited. 


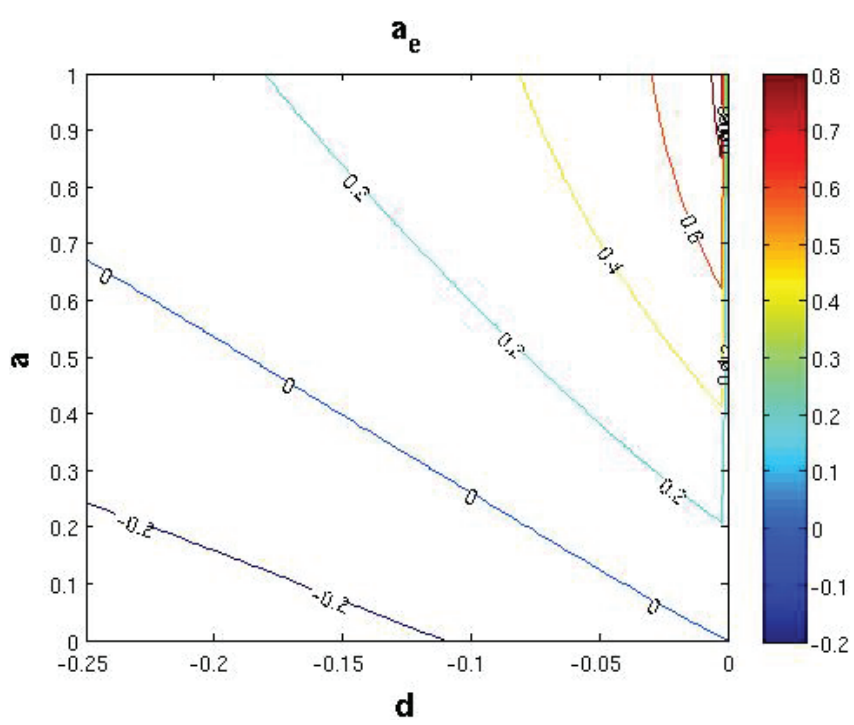

Figure 5. This is the contour plot of $a_{e}$ over the region $(d, a) \subseteq(-1 / 4,0) \times(0,1)$. Note that as $d$ increases to $0, a_{e}$ approaches $a \in(0,1)$.

Since $\alpha \beta<0$, the diffusion-coupling parameters $d_{e}, d_{o}$ are both positive (ellipticity condition). In $(v, w)$-coordinates, the set of equilibria of (4.2) are

$$
E=\left\{(v, w): v=x / \tau_{1}, w=y / \tau_{2}, \text { where }(x, y) \in \hat{E}\right\} .
$$

For parameter values $(d, a)$, where $E$ has nine points, the elements of $E$ are

Case $1(a<0)$.

$$
\begin{aligned}
E=\left\{(0,0),\left(a / \tau_{1}, a / \tau_{2}\right),\left(1 / \tau_{1}, 1 / \tau_{2}\right),(1,1),\left(\tau_{2} / \tau_{1}, \tau_{1} / \tau_{2}\right),\left(a^{-} / \tau_{1}, a^{+} / \tau_{2}\right),\right. \\
\\
\left.\left(a^{+} / \tau_{1}, a^{-} / \tau_{2}\right),\left(1^{-} / \tau_{1}, 1^{+} / \tau_{2}\right),\left(1^{+} / \tau_{1}, 1^{-} / \tau_{2}\right)\right\} .
\end{aligned}
$$

Case $2(0<a<1)$.

$$
\begin{aligned}
E=\left\{(0,0),\left(a / \tau_{1}, a / \tau_{2}\right),\left(1 / \tau_{1}, 1 / \tau_{2}\right),(1,1),\left(\tau_{2} / \tau_{1}, \tau_{1} / \tau_{2}\right),\left(0^{-} / \tau_{1}, 0^{+} / \tau_{2}\right),\right. \\
\\
\left.\left(0^{+} / \tau_{1}, 0^{-} / \tau_{2}\right),\left(1^{-} / \tau_{1}, 1^{+} / \tau_{2}\right),\left(1^{+} / \tau_{1}, 1^{-} / \tau_{2}\right)\right\} .
\end{aligned}
$$

Case $3(a>1)$.

$$
\begin{aligned}
E=\{ & (0,0),\left(a / \tau_{1}, a / \tau_{2}\right),\left(1 / \tau_{1}, 1 / \tau_{2}\right),(1,1),\left(\tau_{2} / \tau_{1}, \tau_{1} / \tau_{2}\right),\left(0^{-} / \tau_{1}, 0^{+} / \tau_{2}\right), \\
& \left.\left(0^{+} / \tau_{1}, 0^{-} / \tau_{2}\right),\left(a^{-} / \tau_{1}, a^{+} / \tau_{2}\right),\left(a^{+} / \tau_{1}, a^{-} / \tau_{2}\right)\right\} .
\end{aligned}
$$

Elements of $E$ that are in the box $(0,1) \times(0,1)$ are called intermediate points of (4.2). For $a<0$, we do not have intermediate points. For $a>0$, the only intermediate point is $\left(0^{+} / \tau_{1}, 0^{-} / \tau_{2}\right)$, which exists only when $a+4 d>0$.

Next, let us investigate the stability of our equilibria. In $(v, w)$-coordinates, the boundary conditions are $\overrightarrow{\mathbf{0}} \equiv\left(\frac{0}{\tau_{1}}, \frac{0}{\tau_{2}}\right), \overrightarrow{\mathbf{1}} \equiv\left(\frac{\tau_{1}}{\tau_{1}}, \frac{\tau_{2}}{\tau_{2}}\right)$ while the intermediate point is $\overrightarrow{\boldsymbol{a}} \equiv\left(\frac{0^{+}}{\tau_{1}}, \frac{0^{-}}{\tau_{2}}\right)$. From the previous section, the stability of $(v, w)=\overrightarrow{\mathbf{0}}, \overrightarrow{\mathbf{1}}, \overrightarrow{\boldsymbol{a}}$ can be inferred from the stability of 
$(x, y)=(0,0),\left(\tau_{1}, \tau_{2}\right),\left(0^{+}, 0^{-}\right)$, respectively. In particular, using the results from the previous section, we have that $\left(\tau_{1}, \tau_{2}\right)$ is stable if $2 d+f_{a}^{\prime}\left(\tau_{1}\right)>0$, which is satisfied when $1-a+2 d>0$. Also, $(0,0)$ is stable (hence, $\overrightarrow{\mathbf{0}}$ is stable) whenever $a+4 d>0$. Finally, $\left(0^{+}, 0^{-}\right)$is unstable if $2 d+f_{a}^{\prime}\left(0^{+}\right)<0$ and exists only when $a+4 d>0$.

Lemma 6.

1. If $a+4 d>0,1-a+2 d>0$, and $2 d+f_{a}^{\prime}\left(0^{+}\right)<0$, then the antidiffusion lattice Nagumo system exhibits bistable dynamics.

2. If $a+4 d<0<1-a+2 d$, then the antidiffusion lattice Nagumo system exhibits monostable dynamics.

Finally, we apply the theorems of Chen, Guo, and $\mathrm{Wu}[7]$ as restated in section 3. In particular, the existence theorem holds for all $(d, a)$.

Lemma 7. There exists a monotone traveling wavefront 2-periodic solution to the system (2.2) that connects $(0,0)$ to $\left(\tau_{1}, \tau_{2}\right)$. If the conditions for bistable dynamics in Lemma 6 hold, then this traveling wavefront solution is unique, 2-periodic, monotonic, and globally exponentially stable.

5.1.2. Propagation failure. In this section, we look at a phenomenon called propagation failure, a distinctive characteristic of LDEs. The first part of this section collects some results on propagation failure in the positive diffusion problem. The basic idea, as presented by Keener [21], is to consider a mapping $\Phi_{K}$ on the plane and to find points on the unit interval that will define some bounded areas in the unit square which will be mapped by $\Phi_{K}$ into themselves (see discussion in [21]). The existence of such points on the unit interval will guarantee the existence of propagation failure in the system.

Our goal is to investigate and derive conditions under which the traveling wavefronts from $(0,0)$ to $\left(\tau_{1}, \tau_{2}\right)$ to the antidiffusion lattice Nagumo system with bistable dynamics fail to propagate. To this aim, we will, initially, look at the limiting equations of (4.2) to find an approximation $\hat{\Phi}$ to a map $\Phi$ that is analogous to Keener's mapping $\Phi_{K}$. We use an approximation $\hat{\Phi}$ because the mapping $\Phi$ is of degree 9 and is complicated to analyze directly. This section ends with a result that contains sufficient conditions such that traveling waves connecting $(0,0)$ to $\left(\tau_{1}, \tau_{2}\right)$ fail to propagate.

Positive diffusion. In the reaction-diffusion PDE case, for example,

$$
u_{t}=d u_{x x}-f_{a}(u), \quad x, t \in \mathbb{R}, \quad d>0,
$$

the wave speed $c$ of the traveling wave solution, if it exists, is a continuous and strictly monotonic function of $a$ (that is, no propagation failure). In the reaction-diffusion PDE case with spatially periodic coefficients, for example,

$$
\partial_{t} u=d \partial_{x}\left(b(x) \partial_{x} u\right)+f_{a}(u), \quad x, t \in \mathbb{R}, \quad d>0,
$$

with $b(x)=b(x+2 \pi)$, stationary solutions $c=0$ solve a time-periodic ODE. Heteroclinic orbits are now typically transverse, so that we expect pinned fronts for an interval of values of $a$.

In the LDE case,

$$
\dot{u}_{n}(t)=d\left(u_{n+1}-2 u_{n}+u_{n-1}\right)-f_{a}\left(u_{n}\right), \quad d>0,
$$

Copyright $\odot$ by SIAM. Unauthorized reproduction of this article is prohibited. 


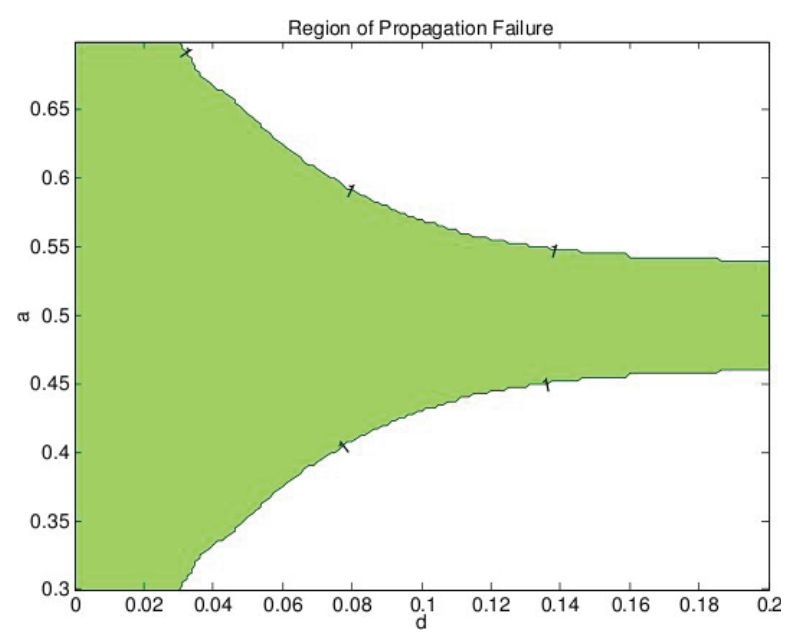

Figure 6. The shaded area shows the region of propagation failure for the positive diffusion problem in the $(d, a)$-parameter plane.

where $f_{a}(u)=u(u-a)(u-1)$ with $0<a<1$, the wave speed of the traveling wave solution may be zero for an open set of $a$. In particular, for sufficiently small $d>0$, there are numbers $a_{-} \neq a_{+}$in the interval $(0,1)$ such that $c=c(a)$ satisfies

$$
c\left\{\begin{array}{l}
<0, \quad 0<a<a_{-}, \\
=0, \quad a_{-} \leq a \leq a_{+}, \\
>0, \quad a_{+}<a<1 .
\end{array}\right.
$$

When the numbers $a_{-} \neq a_{+}$exist, we say that pinning of the wave or propagation failure occurs; the wave is pinned and cannot propagate when $a$ is in the nontrivial interval $\left[a_{-}, a_{+}\right]$. This interval is usually called the pinning interval, and the length of the interval gives a measure of the pinning of the waves.

A numerical approximation of the region of propagation failure for the positive diffusion problem is shown in Figure 6. This was obtained (as was Figure 7 in a similar way) by starting from a Heaviside initial condition, approximating the solution of the differential equations using the MATLAB code ODE45 with tolerances of $10^{-10}$ to a final time of $T=100$. If

$$
u_{-1}(100)=w_{-1}(100)>0.95 \quad \text { or } \quad u_{0}(100)=v_{0}(100)<0.05
$$

the parameter value in the $(d, a)$-plane was deemed to not have propagation failure; otherwise it was labeled as having propagation failure.

To obtain a qualitative picture of the region of propagation failure, we analyze the range of existence of monotonic steady-state solutions, as argued by Keener in [21]. He proved the existence of a pinning interval by applying a result due to Moser. He showed that the mapping $\phi_{f_{a}, d}$ defined by

$$
\phi_{f_{a}, d}(u, v)=\left(\frac{f_{a}(u)}{d}+2 u-v, u\right)
$$

Copyright (c) by SIAM. Unauthorized reproduction of this article is prohibited. 


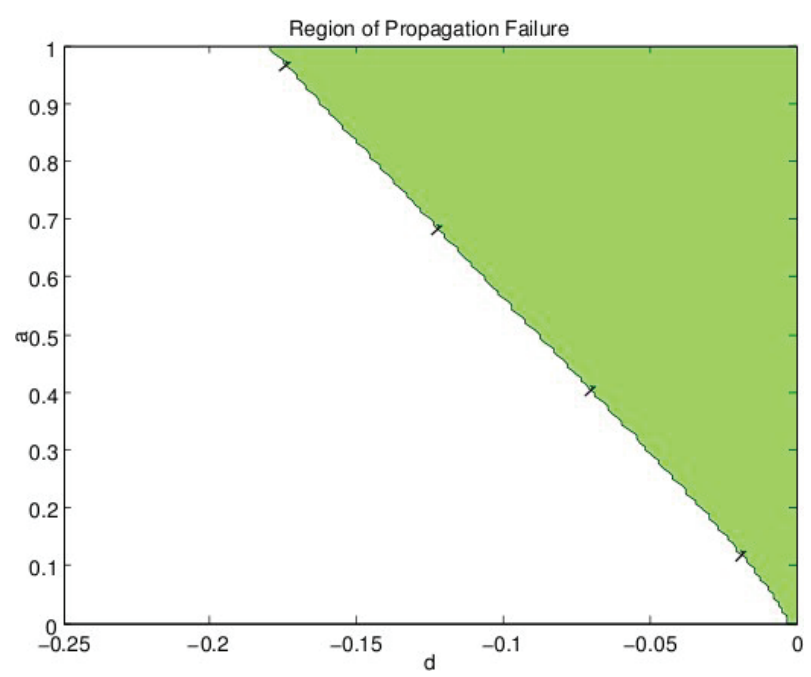

Figure 7. The shaded area shows the region of propagation failure for the antidiffusion lattice Nagumo problem in a bistable region $B$.

possesses symbolic dynamics (on two symbols). This mapping is obtained by setting $\dot{u}_{n}(t)=0$ in (5.1) and writing the resulting difference equation

$$
d\left(u_{n+1}-2 u_{n}+u_{n-1}\right)-f_{a}\left(u_{n}\right)=0, \quad d>0,
$$

as a map on the plane by taking

$$
u_{n+1}=\frac{f_{a}\left(u_{n}\right)}{d}+2 u_{n}-v_{n}, \quad v_{n+1}=u_{n} .
$$

For the cubic-nonlinearity $f_{a}(u)=u(u-a)(u-1)$, where $0<a<1 / 2$, Keener derived a pinning interval

$$
\sqrt{4 d}<a<1-\sqrt{4 d}, \quad 0 \leq d \leq 1 / 16 .
$$

Negative diffusion. We want to know if the propagation failure phenomenon exists in the antidiffusion lattice Nagumo traveling wavefront problem in a bistable region $B$ for the connection $(0,0)$ to $\left(\tau_{1}, \tau_{2}\right)$. From Lemma $6, B$ is a subset of $\{a+4 d>0,1-a+2 d>$ $\left.0,2 d+f_{a}^{\prime}\left(0^{+}\right)<0\right\}$, where $\left(0^{+}, 0^{-}\right)$is the only intermediate point (for $\left.a>0\right)$. In this part, we will assume that $0<a<1$ (similar arguments apply for the other case, $a>1$ ). Thus, consider the bistable region

$$
B=\left\{(d, a): a+4 d>0,1-a+2 d>0,2 d+f_{a}^{\prime}\left(0^{+}\right)<0,0<a<1\right\} ;
$$

that is, if $(d, a) \in B$, then both $(v, w)=(0,0)$ and $(v, w)=(1,1)$ are stable equilibria to $(4.2)$ and the only other intermediate point is $(v, w)=\left(\frac{0^{+}}{\tau_{1}}, \frac{0^{-}}{\tau_{2}}\right)$, which is unstable.

Consider $\dot{u}_{n}=F_{n}\left(u_{n-1}, u_{n}, u_{n+1}\right)$, where

$$
F_{n}\left(u_{n-1}, u_{n}, u_{n+1}\right)=d_{n}\left(u_{n+1}-2 u_{n}+u_{n-1}\right)-f_{n}\left(u_{n}\right), \quad n \in \mathbb{Z},
$$

Copyright $\odot$ by SIAM. Unauthorized reproduction of this article is prohibited. 
where $\left(d_{n}, f_{n}\right)=\left(d_{e}, f_{e}\right)$ for $n$ an even number and $\left(d_{n}, f_{n}\right)=\left(d_{o}, f_{o}\right)$ for $n$ an odd number. Setting $\dot{u}_{n}=0$ and then solving for $u_{n+1}$, we have

$$
u_{n+1}=\frac{f_{n}\left(u_{n}\right)}{d_{n}}+2 u_{n}-v_{n}, \quad v_{n}=u_{n-1} .
$$

Define the following maps:

$$
\begin{gathered}
\phi_{e}(u, v)=\left(\frac{f_{e}(u)}{d_{e}}+2 u-v, u\right), \quad \phi_{o}(u, v)=\left(\frac{f_{o}(u)}{d_{o}}+2 u-v, u\right), \\
\Phi=\phi_{o} \circ \phi_{e} .
\end{gathered}
$$

The mapping $\Phi$ is of degree 9 , and our goal is to study the mapping $\Phi$, which we can view as iterating the mapping (5.3) twice. The explicit expression for $\Phi$ is

$$
\Phi(u, v)=\left(\frac{f_{o}\left(\frac{f_{e}(u)}{d_{e}}+2 u-v\right)}{d_{o}}+\frac{2 f_{e}(u)}{d_{e}}+3 u-v, \frac{f_{e}(u)}{d_{e}}+2 u-v\right) .
$$

We want to derive sufficient conditions for $d$ and $a$ in region $B$ that give rise to propagation failure. In $B$, the detuning parameter $a_{e}$ for the even nodes satisfies $0<a_{e}<1$, and so we can view $\phi_{e}$ as the mapping $\phi_{f_{e}, d_{e}}$ in (5.3). However, the detuning parameter $a_{o}$ for the odd nodes satisfies $a_{o}<-1$, and so we cannot apply an analysis similar to that in the even nodes. Instead, we will use an approximation of $\phi_{o}$. To obtain such an approximation, let us study how the system (4.2) behaves as $d \rightarrow 0-$.

Lemma 8. Consider the connection $(0,0)$ to $\left(\tau_{1}, \tau_{2}\right)$ for a fixed $(d, a)$ in a bistable region B. The equations (4.2) have limits

$$
\dot{v}_{j}=-f_{a}\left(v_{j}\right), \quad \dot{w}_{j}=\frac{a}{2}\left(v_{j+1}-2 w_{j}+v_{j}\right)
$$

as $d \rightarrow 0-$.

Proof. To see this, start from (4.2):

$$
\left\{\begin{array}{c}
\dot{v}_{j}=d_{e}\left(w_{j}-2 v_{j}+w_{j-1}\right)-f_{e}\left(v_{j}\right), \\
\dot{w}_{j}=d_{o}\left(v_{j+1}-2 w_{j}+v_{j}\right)-f_{o}\left(w_{j}\right),
\end{array} \quad j \in \mathbb{Z} .\right.
$$

Since $\tau_{1} \rightarrow 1+$ and $\tau_{2} \rightarrow 0-$ as $d \rightarrow 0-$, we clearly have that $d_{e} \rightarrow 0+$ and $f_{e}(u) \rightarrow f_{a}(u)$ (because $a_{e} \rightarrow a$ ) as $d \rightarrow 0-$. Furthermore,

$$
f_{o}(u)=\tau_{2}^{2} u(u-1)\left(u-a_{o}\right)=\tau_{2} u(u-1)\left(\tau_{2} u-\left(a+1-\tau_{2}\right)\right)
$$

so that $f_{o}(u) \rightarrow 0$ as $d \rightarrow 0-$.

Next, we wish to show that $d_{o} \rightarrow a / 2$ as $d \rightarrow 0-$. To see this we will consider, for $a$ fixed, $\tau_{1} \equiv \tau_{1}(d)$ and $\tau_{2} \equiv \tau_{2}(d)$. We obtain

$$
\lim _{d \rightarrow 0-} d_{o}=\lim _{d \rightarrow 0-} d \frac{\tau_{1}(d)}{\tau_{2}(d)}=\lim _{d \rightarrow 0-} \frac{d \tau_{1}^{\prime}(d)+\tau_{1}(d)}{\tau_{2}^{\prime}(d)}
$$

Copyright $\odot$ by SIAM. Unauthorized reproduction of this article is prohibited. 
after applying L'Hôpital's rule. To evaluate $\tau_{1}^{\prime}(d)$ and $\tau_{2}^{\prime}(d)$ we differentiate the equations

$$
f_{a}\left(\tau_{1}(d)\right)+f_{a}\left(\tau_{2}(d)\right)=0, \quad 2 d\left(\tau_{1}(d)-\tau_{2}(d)\right)=f_{a}\left(\tau_{2}(d)\right)
$$

with respect to $d$ to obtain the linear system

$$
\left(\begin{array}{cc}
f_{a}^{\prime}\left(\tau_{1}\right) & f_{a}^{\prime}\left(\tau_{2}\right) \\
-2 d & 2 d+f_{a}^{\prime}\left(\tau_{2}\right)
\end{array}\right)\left(\begin{array}{c}
\tau_{1}^{\prime} \\
\tau_{2}^{\prime}
\end{array}\right)=\left(\begin{array}{c}
0 \\
2\left(\tau_{1}-\tau_{2}\right)
\end{array}\right)
$$

In the limit as $d \rightarrow 0-$ we have

$$
\left(\begin{array}{cc}
1-a & a \\
0 & a
\end{array}\right),\left(\begin{array}{c}
\tau_{1}^{\prime}(0-) \\
\tau_{2}^{\prime}(0-)
\end{array}\right)=\left(\begin{array}{l}
0 \\
2
\end{array}\right)
$$

which has solution $\tau_{1}^{\prime}(0-)=-2 /(1-a)$ and $\tau_{2}^{\prime}(0-)=2 / a$. Thus,

$$
\lim _{d \rightarrow 0-} d_{o}=\lim _{d \rightarrow 0-} \frac{d \tau_{1}^{\prime}(d)+\tau_{1}(d)}{\tau_{2}^{\prime}(d)}=\frac{1}{2 / a}=a / 2 .
$$

Setting $\dot{v}_{j}=\dot{w}_{j}=0$, the limiting equations (5.8) yield $v_{j}=0, a, 1(a \in(0,1))$ and $w_{j}=\frac{1}{2}\left(v_{j}+v_{j+1}\right)$. This implies that if, for example,

$$
\overrightarrow{\boldsymbol{v}}=\{\ldots, 0, \ldots, 0,0, a, \ldots, a, a, 1,1 \ldots\}, \text { then } \overrightarrow{\boldsymbol{w}}=\left\{\ldots, 0, \ldots, 0, \frac{a}{2}, a, \ldots, a, \frac{a+1}{2}, 1, \ldots\right\}
$$

or if

$$
\overrightarrow{\boldsymbol{v}}=\{\ldots, 0, \ldots, 0,0,1,1 \ldots\}, \text { then } \overrightarrow{\boldsymbol{w}}=\{\ldots, 0, \ldots, 0,1 / 2,1, \ldots\} .
$$

These pairs $(\overrightarrow{\boldsymbol{v}}, \overrightarrow{\boldsymbol{w}})$ form monotone standing wave solutions $(c=0)$ to the limiting equations that connect $\left(v_{-}, w_{-}\right)=(0,0)$ to $\left(v_{+}, w_{+}\right)=(1,1)$. Other monotone solutions can be obtained by varying the location and the number of $a$ 's in the middle of $\overrightarrow{\boldsymbol{v}}$.

Approximating the mapping $\boldsymbol{\Phi}$. Applying the analysis in Lemma 7 (in particular, the fact that $f_{o} \rightarrow 0$ as $d \rightarrow 0-$ ), we will initially approximate $\phi_{o}$ in (5.6) by the linear map

$$
\phi_{L}(u, v)=(2 u-v, u)
$$

Hence, consider the map $\hat{\Phi}$ defined by

$$
\hat{\Phi}(u, v)=\phi_{L} \circ \phi_{e}(u, v)=\left(\frac{2}{d_{e}} f_{e}(u)+3 u-2 v, \frac{1}{d_{e}} f_{e}(u)+2 u-v\right) .
$$

Note that the inverses of $\phi_{e}, \phi_{L}$, respectively, are

$$
\phi_{e}^{-1}(u, v)=\left(v, \frac{1}{d_{e}} f_{e}(u)+2 v-u\right), \quad \phi_{L}^{-1}(u, v)=(v, 2 v-u)
$$

so that

$$
\hat{\Phi}^{-1}(u, v)=\phi_{e}^{-1} \circ \phi_{L}^{-1}(u, v)=\left(2 v-u, \frac{1}{d_{e}} f_{e}(2 v-u)+3 v-2 u\right) .
$$

Copyright (C by SIAM. Unauthorized reproduction of this article is prohibited. 
The main idea of the proof of the next lemma lies on the fact that the mapping $\hat{\Phi}$ is a homeomorphism on the unit square $[0,1] \times[0,1]$ into the plane $\mathbb{R}^{2}$. This next lemma is analogous to Keener's Corollary 2.2 on page 560 of [21]. Our result is the following lemma.

Lemma 9. Suppose there are numbers $\hat{u}_{j} \in(0,1)$ and $\hat{u}_{j}^{*} \in(0,1)$ for $j \in\{0,1,2, \ldots, 5\}$ such that the following hold:

1. $H_{-1}\left(\hat{u}_{0}\right)=H_{-2}\left(\hat{u}_{1}\right)=H_{-3}\left(\hat{u}_{2}\right)=H_{0}\left(\hat{u}_{3}\right)=H_{-1}\left(\hat{u}_{4}\right)=H_{-2}\left(\hat{u}_{5}\right)=0$, where

$$
H_{-k}(u)=\frac{2}{d_{e}} f_{e}(u)+3 u-k \quad \text { for } \quad k \in\{0,1,2,3\} ;
$$

2.

$$
\hat{u}_{j}^{*}= \begin{cases}\frac{1}{d_{e}} f_{e}\left(\hat{u}_{j}\right)+2 \hat{u}_{j}, & j=0,3,4, \\ \frac{1}{d_{e}} f_{e}\left(\hat{u}_{j}\right)+2 \hat{u}_{j}-1, & j=1,2,5 .\end{cases}
$$

3.

$$
\frac{2}{d_{e}} f_{e}^{\prime}(u)+3>0 \text { for } \begin{cases}0 \leq u \leq \hat{u}_{j}, & j=0,1,2 \\ \hat{u}_{j} \leq u \leq 1, & j=3,4,5\end{cases}
$$

Then $\hat{\Phi}$ has

1. a countable infinity of periodic orbits of arbitrarily high period,

2. an uncountable infinity of nonperiodic orbit, and

3. a dense orbit.

Proof. To prove Lemma 9, we need to show that $\hat{\Phi}$ is a Smale-horseshoe type of mapping and as such, it will be topologically conjugate with the shift mapping $\sigma$ on the space $\Sigma$ of bi-infinite sequences on two symbols [26]. Geometrically, the mapping $\hat{\Phi}$ contracts the vertical direction, expands the horizontal direction, and folds the unit square around, laying it back on itself while fixing the points $(0,0)$ and $(1,1)$, in such a way that we can find disjoint regions that are mapped over themselves. A Smale-horseshoe mapping need not have the shape of a horseshoe (an inverted U); in fact, for our problem, the mapping $\hat{\Phi}$ yields a G-shaped horseshoe, as sketched in Figure 8.

In Figures 8 and 9, we use the following notation to identify points:

$$
\begin{array}{ll}
U_{j}=\left(\hat{u}_{j}, 0\right), j=0,3,4 ; & U_{j}=\left(\hat{u}_{j}, 1\right), j=1,2,5 ; \\
U_{j}^{*}=\left(0, \hat{u}_{j}^{*}\right), j=1,3,5 ; & U_{j}^{*}=\left(1, \hat{u}_{j}^{*}\right), j=0,2,4 .
\end{array}
$$

To show that $\hat{\Phi}$ is a Smale-horseshoe type of mapping, we will apply the so-called ConleyMoser conditions [26] (see Theorem 25.1.5). For our problem, these conditions translate to finding 12 points $U_{j}, U_{j}^{*}$ for $j=0,1,2,3,4,5$ such that the following hold:

1. $\hat{\Phi}\left(U_{j}\right)=U_{j}^{*}$ for $j=0,1,2,3,4,5$.

2. The points $O, U_{0}, U_{2}, U_{1}$ define a vertical strip $V_{0}$. The points $U_{3}, U_{4}, P, U_{5}$ define a vertical strip $V_{1}$. The two strips $V_{0}, V_{1}$ are disjoint.

3. The points $O, U_{0}^{*}, U_{2}^{*}, U_{1}^{*}$ define the horizontal strip $H_{0}$ while the points $U_{3}^{*}, U_{4}^{*}, P, U_{5}^{*}$ define the horizontal strip $H_{1}$. The two strips $H_{0}, H_{1}$ are disjoint.

4. $\hat{\Phi}\left(V_{i}\right)=H_{i}$ for $i=0,1$. 


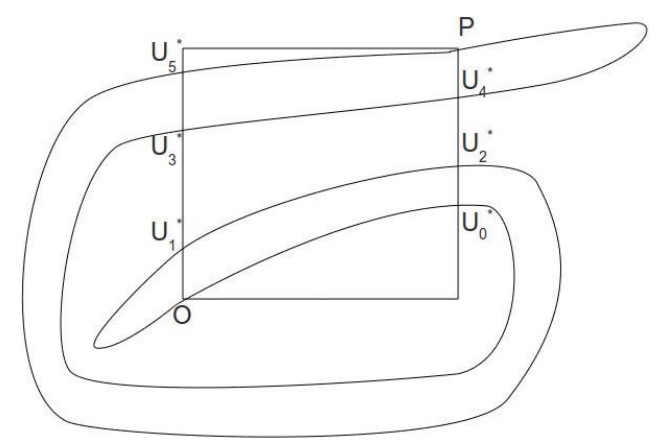

Figure 8. The diagram shows a sketch of the image of the unit square under the mapping $\hat{\Phi}$.

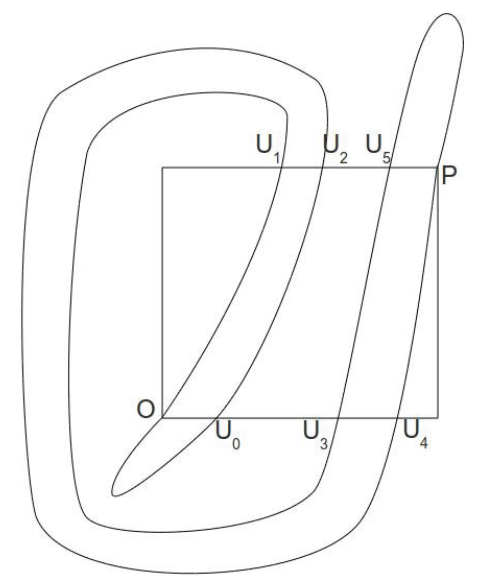

Figure 9. The diagram shows a sketch of the image of the unit square under the inverse mapping $\hat{\Phi}^{-1}$.

5. The horizontal boundary curves of $V_{i}$ map to the horizontal boundary curves of $H_{i}$ and the vertical boundary curves of $V_{i}$ map to the vertical boundary curves of $H_{i}$.

A horizontal strip is the set lying between two nonintersecting horizontal curves; a vertical strip is the set lying between two nonintersecting vertical curves. A horizontal curve $v=v(u)$ is the graph of a function $0 \leq v(u) \leq 1$ where $u \in[0,1]$; a vertical curve $u=u(v)$ is the graph of a function $0 \leq v(u) \leq 1$ where $u \in[0,1]$. Figure 10 illustrates the points $U_{j}, U_{j}^{*}$ and the strips $V_{i}, H_{i}$ in one unit square. Note that the boundary curves need not be straight lines.

The following table summarizes the mapping of the points and the conditions that the points $\hat{u}_{j}, \hat{u}_{j}^{*}$ must satisfy:

\begin{tabular}{|l|l|l|}
\hline$\hat{\Phi}\left(U_{0}\right)=U_{0}^{*}$ & $\frac{2}{d_{e}} f_{e}\left(\hat{u}_{0}\right)+3 \hat{u}_{0}=1$ & $\hat{u}_{0}^{*}=\frac{1}{d_{e}} f_{e}\left(\hat{u}_{0}\right)+2 \hat{u}_{0}$ \\
\hline$\hat{\Phi}\left(U_{1}\right)=U_{1}^{*}$ & $\frac{2}{d_{e}} f_{e}\left(\hat{u}_{1}\right)+3 \hat{u}_{1}-2=0$ & $\hat{u}_{1}^{*}=\frac{1}{d_{e}} f_{e}\left(\hat{u}_{1}\right)+2 \hat{u}_{1}-1$ \\
\hline$\hat{\Phi}\left(U_{2}\right)=U_{2}^{*}$ & $\frac{2}{d_{e}} f_{e}\left(\hat{u}_{2}\right)+3 \hat{u}_{2}-2=1$ & $\hat{u}_{2}^{*}=\frac{1}{d_{e}} f_{e}\left(\hat{u}_{2}\right)+2 \hat{u}_{2}-1$ \\
\hline$\hat{\Phi}\left(U_{3}\right)=U_{3}^{*}$ & $\frac{2}{d_{e}} f_{e}\left(\hat{u}_{3}\right)+3 \hat{u}_{3}=0$ & $\hat{u}_{3}^{*}=\frac{1}{d_{e}} f_{e}\left(\hat{u}_{3}\right)+2 \hat{u}_{3}$ \\
\hline$\hat{\Phi}\left(U_{4}\right)=U_{4}^{*}$ & $\frac{2}{d_{e}} f_{e}\left(\hat{u}_{4}\right)+3 \hat{u}_{4}=1$ & $\hat{u}_{4}^{*}=\frac{1}{d_{e}} f_{e}\left(\hat{u}_{4}\right)+2 \hat{u}_{4}$ \\
\hline$\hat{\Phi}\left(U_{5}\right)=U_{5}^{*}$ & $\frac{2}{d_{e}} f_{e}\left(\hat{u}_{5}\right)+3 \hat{u}_{5}-2=0$ & $\hat{u}_{5}^{*}=\frac{1}{d_{e}} f_{e}\left(\hat{u}_{5}\right)+2 \hat{u}_{5}-1$ \\
\hline
\end{tabular}

Copyright (C) by SIAM. Unauthorized reproduction of this article is prohibited. 


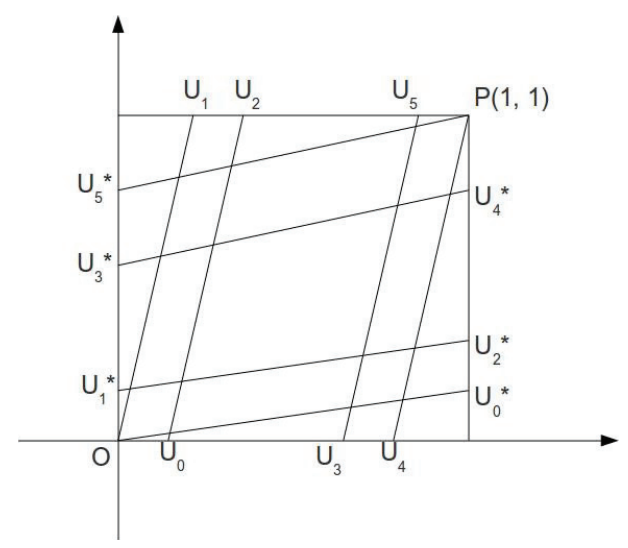

Figure 10. The diagram shows a sketch of the 12 points that we are looking for and the vertical and horizontal strips.

Thus, if we define

$$
H_{-k}(u)=\frac{2}{d_{e}} f_{e}(u)+3 u-k \quad \text { for } \quad k \in\{0,1,2,3\},
$$

then the second column of the table gives the following conditions on the points $\hat{u}_{j}$ :

$$
H_{-1}\left(\hat{u}_{0}\right)=H_{-2}\left(\hat{u}_{1}\right)=H_{-3}\left(\hat{u}_{2}\right)=H_{0}\left(\hat{u}_{3}\right)=H_{-1}\left(\hat{u}_{4}\right)=H_{-2}\left(\hat{u}_{5}\right)=0
$$

while the third column of the table gives the following conditions on the points $u_{j}^{*}$ :

$$
\hat{u}_{j}^{*}= \begin{cases}\frac{1}{d_{e}} f_{e}\left(\hat{u}_{j}\right)+2 \hat{u}_{j}, & j=0,3,4, \\ \frac{1}{d_{e}} f_{e}\left(\hat{u}_{j}\right)+2 \hat{u}_{j}-1, & j=1,2,5 .\end{cases}
$$

For the two vertical strips $V_{i}$ to be disjoint, we require that $\hat{u}_{2}<\hat{u}_{3}$; for the two horizontal strips $H_{i}$ to be disjoint, we require that $\hat{u}_{3}^{*}<\hat{u}_{2}^{*}$. Observe that there is a slight abuse of notation: $H_{0}$ may refer to the polynomial $H_{k}$ for $k=0$ or may refer to the horizontal strip $H_{0}$; the context should indicate which notion we are looking at. Finally, to prove that the boundary curves of the strips behave in a required manner, one applies arguments similar to Keener's proof [21].

There is a linear relationship between $\hat{u}_{j}$ and $\hat{u}_{j}^{*}$ for each $j$ as follows:

$$
\hat{u}_{j}^{*}=\frac{1}{2} \hat{u}_{j}, \quad j \in\{1,3,5\}, \quad \hat{u}_{j}^{*}=\frac{1}{2}\left(\hat{u}_{j}+1\right), \quad j \in\{0,2,4\} .
$$

Remark 1. The first two requirements on $\hat{u}_{j}, \hat{u}_{j}^{*}$ of Lemma 9 guarantee the existence of horizontal strips $H_{i}$ and vertical strips $V_{i}$ with $\hat{\Phi}\left(V_{i}\right)=H_{i}$ for $i=0,1$ that satisfy the Conley-Moser conditions (in a weaker form). The third condition of Lemma 9 guarantees that the the boundary curves of these strips are monotone increasing. 
The next result contains sufficient conditions on the parameters $(d, a)$ in a bistable region $B$ that will imply the conditions of Lemma 9 , and hence $\hat{\Phi}$ will be topologically conjugate to the shift mapping on two symbols $\{0,1\}$. Lemma 9 guarantees that for any sequence $\left\{s_{j}\right\}_{j \in \mathbb{N}}$ with $s_{j} \in\{0,1\}$, there is a sequence $\left\{\left(v_{j}, w_{j}\right)\right\}_{j \in \mathbb{N}}$ which is an equilibrium solution of

$$
\left\{\begin{array}{l}
\dot{v}_{j}=d_{e}\left(w_{j}-2 v_{j}+w_{j-1}\right)-f_{e}\left(v_{j}\right), \quad j \in \mathbb{Z}, \\
\dot{w}_{j}=d_{o}\left(v_{j+1}-2 w_{j}+v_{j}\right),
\end{array}\right.
$$

with

$$
\begin{cases}\left(v_{j}, w_{j}\right) \in\left[0, \hat{u}_{2}\right] \times\left[0, \hat{u}_{2}^{*}\right], & s_{j}=0 \\ \left(v_{j}, w_{j}\right) \in\left[\hat{u}_{3}, 1\right] \times\left[\hat{u}_{3}^{*}, 1\right], & s_{j}=1 .\end{cases}
$$

Equivalently, the next lemma will imply that the system (5.17) will have traveling wave solutions with zero speed, the propagation failure phenomenon.

Lemma 10. Consider the connection $(0,0)$ to $\left(\tau_{1}, \tau_{2}\right)$ for a fixed $(d, a)$ in a bistable region B. Suppose

$$
\frac{1}{\tau_{1}}\left(\tau_{1}^{2}+\sqrt{6 d \tau_{2} \tau_{1}}-\tau_{1}\right) \leq a \leq \frac{1}{\tau_{1}}\left(2 \tau_{1}^{2}-\sqrt{6 d \tau_{2} \tau_{1}}-\tau_{1}\right) .
$$

Then $\hat{\Phi}$ possesses the shift on sequences on two symbols as a subsystem.

Proof. The previous lemma claims that the existence of $\left\{\hat{u}_{j}\right\},\left\{\hat{u}_{j}^{*}\right\}$ for $j=0,1, \ldots, 5$ in the unit interval guarantees that there is propagation failure. Let us find a relationship between $d_{e}$ and $a_{e}$ that implies the hypotheses of this lemma. Observe that the equations satisfied by $\hat{u}_{j}$ in (5.15) are translates of each other. Conditions for the existence of roots of $H_{0}$ and $H_{-3}$ can be computed because $u$ is a factor of $H_{0}$ while $u-1$ is a factor of $H_{-3}$, and hence the two equations $H_{0}(u)=0=H_{-3}(u)$ are quadratic. Now, even without explicitly computing the other four roots $\hat{u}_{k}$ for $k=0,1,4,5$, which are roots of a cubic equation with positive discriminant, we can still ensure that these exist because these four roots are bounded by $0, \hat{u}_{2}, \hat{u}_{3}, 1$. In particular, the smallest root of $H_{0}$, which is 0 , bounds the roots of the $H_{-k}$ 's on the left while the largest root of $H_{-3}$, which is 1 , bounds the roots of $H_{-k}$ 's on the right. This is based on the observation that the $H_{-k}$ 's for $k=1,2,3,4,5$ are just vertical translations of $H_{0}$. Thus, the existence of three distinct real roots of $H_{0}$ and $H_{-3}$ will imply the existence of the roots of $H_{-1}$ and $H_{-2}$.

For example, if $a=3 / 4$ and $d=-1 / 8$, then $\left(\tau_{1}, a_{e}\right) \approx(1.31383,0.331979)$. The four curves $H_{-k}(u)$ for $k \in\{0,1,2,3\}$ are illustrated in Figure 11 .

To guarantee the existence of the roots of $H_{0}$ and $H_{-3}$, since they are quadratic expressions, the discriminant of the quadratic equation $H_{0}=0=H_{-3}$ must be nonnegative, that is,

$$
\left(a_{e}+1\right)^{2}-4 a_{e}-\frac{6 d_{e}}{\tau_{1}^{2}} \geq 0, \quad a_{e}^{2}-\frac{6 d_{e}}{\tau_{1}^{2}} \geq 0
$$

for $H_{0}, H_{-3}$, respectively. These inequalities are equivalent to

$$
d_{e} \leq \frac{1}{6} \tau_{1}^{2} a_{e}^{2}, \quad d_{e} \leq \frac{1}{6} \tau_{1}^{2}\left(a_{e}-1\right)^{2},
$$

respectively. In other words, the hypotheses of Lemma 9 will hold whenever

$$
d_{e}=\min \left\{\begin{array}{ll}
\frac{1}{6} \tau_{1}^{2} a_{e}^{2}, & \frac{1}{6} \tau_{1}^{2}\left(a_{e}-1\right)^{2}
\end{array}\right\}= \begin{cases}\frac{1}{6} \tau_{1}^{2} a_{e}^{2}, & 0<a_{e}<1 / 2, \\
\frac{1}{6} \tau_{1}^{2}\left(a_{e}-1\right)^{2}, & 1 / 2<a_{e}<1 .\end{cases}
$$

Copyright (C) by SIAM. Unauthorized reproduction of this article is prohibited. 


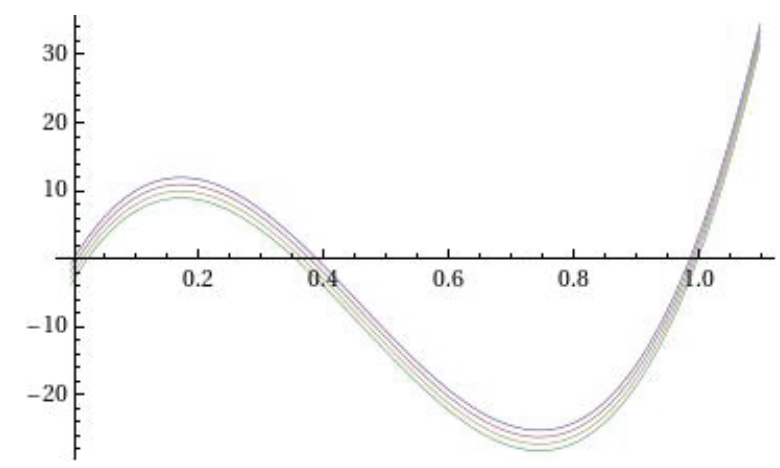

Figure 11. Set $(d, a)=(-1 / 8,3 / 4)$. The translates $H_{-k}(u)$ are sketched here, where the horizontal axis is $u$.

Note that we do not allow $a_{e}=1 / 2$ because in this case, $\hat{u}_{2}=\hat{u}_{3}$, and so we would not have disjoint vertical strips $V_{0}, V_{1}$. Moreover, inequalities in (5.18) imply that

$$
\frac{\sqrt{6 d_{e}}}{\tau_{1}} \leq a_{e} \leq 1-\frac{\sqrt{6 d_{e}}}{\tau_{1}}
$$

which can also be stated in terms of $d$ and $a$ :

$$
\frac{1}{\tau_{1}}\left(\tau_{1}^{2}+\sqrt{6 d \tau_{2} \tau_{1}}-\tau_{1}\right) \leq a \leq \frac{1}{\tau_{1}}\left(2 \tau_{1}^{2}-\sqrt{6 d \tau_{2} \tau_{1}}-\tau_{1}\right) .
$$

Compare the pinning interval in the positive diffusion case (5.4) with the above inequalities (5.19), (5.20). Observe that in (5.20), we have that $0<a<1$ as $d \rightarrow 0-$. These conditions on $d$ and $a$ can be further weakened by considering monotone functions, similar to what Keener did in the positive diffusion case [21].

Out of the two nonzero roots of $H_{0}$, we take the larger root as $\hat{u}_{3}$ :

$$
\hat{u}_{3}=(1 / 2)\left(\left(a_{e}+1\right)+\sqrt{\left(a_{e}-1\right)^{2}-\frac{6 d_{e}}{\tau_{1}^{2}}}\right) ;
$$

while we take the smallest root of $H_{-3}$ as $\hat{u}_{2}$ :

$$
\hat{u}_{2}=(1 / 2)\left(a_{e}-\sqrt{a_{e}^{2}-\frac{6 d_{e}}{\tau_{1}^{2}}}\right) .
$$

We can, in fact, determine the precise ordering of all six numbers $\hat{u}_{j}, j \in\{0,1, \ldots, 5\}$. By monotonicity of the boundary curves of the horizontal and vertical strips, these numbers $\hat{u}_{j}$ in the unit interval must lie outside the spinodal interval $S=\left(s_{-}, s_{+}\right)$of the polynomial $H_{-k}$. The roots $\hat{u}_{0}<\hat{u}_{1}<\hat{u}_{2}$ are the minimum roots of $H_{-1}, H_{-2}, H_{-3}$, respectively, while the roots $\hat{u}_{3}<\hat{u}_{4}<\hat{u}_{5}$ are the maximum roots of $H_{0}, H_{-1}, H_{-2}$; that is, we have the ordering

$$
0<\hat{u}_{0}<\hat{u}_{1}<\hat{u}_{2}<s_{-}<s_{+}<\hat{u}_{3}<\hat{u}_{4}<\hat{u}_{5}<1 .
$$

However, there is no such ordering for $\hat{u}_{j}^{*}$ because of the folding mechanism in $\hat{\Phi},(5.16)$ :

$$
0<\hat{u}_{1}^{*}<\hat{u}_{3}^{*}<\min \left\{\hat{u}_{0}^{*}, \hat{u}_{5}^{*}\right\}<\max \left\{\hat{u}_{0}^{*}, \hat{u}_{5}^{*}\right\}<\hat{u}_{2}^{*}<\hat{u}_{4}^{*}<1 .
$$

Copyright (C) by SIAM. Unauthorized reproduction of this article is prohibited. 
The mapping $\Phi$. Gaining insight from the approximation mapping $\hat{\Phi}$, we now look at the full mapping $\Phi=\phi_{o} \circ \phi_{e}$. Note that $\Phi=\hat{\Phi}+E$, where $E$ is the mapping on the plane defined by

$$
E(u, v)=\left(\frac{1}{d_{o}} f_{o}\left(\frac{1}{d_{e}} f_{e}(u)+2 u-v\right), 0\right) .
$$

Certainly, other perturbations $\hat{\Phi}$ may be used to approximate $\Phi$. We have chosen to approximate $\phi_{o}$ by the linear mapping $\phi_{L}$ because, as seen in the previous discussion, it gave rise to a simpler $\hat{\Phi}$. In particular, the definition of $\hat{\Phi}$ involves the even components $d_{e}, f_{e}, a_{e}$ while the definition of $E$ involves the odd components $d_{o}, f_{o}, a_{o}$. Separating the even from the odd contributions is helpful because the even detuning parameter $a_{e}$ is in the unit interval $(0,1)$ while the odd detuning parameter $a_{o}$ is negative.

A result similar to Lemma 9 is the following lemma. that

Lemma 11. Suppose there are numbers $u_{j} \in(0,1), u_{j}^{*} \in(0,1)$ for $j \in\{0,1,2, \ldots, 5\}$ such 1.

$$
u_{j}= \begin{cases}\frac{1}{d_{o}} f_{o}\left(u_{j}^{*}\right)+2 u_{j}^{*}, & j=1,3,5, \\ \frac{1}{d_{e}} f_{o}\left(u_{j}^{*}\right)+2 u_{j}^{*}-1, & j=0,2,4\end{cases}
$$

2.

$$
u_{j}^{*}= \begin{cases}\frac{1}{d_{e}} f_{e}\left(u_{j}\right)+2 u_{j}, & j=0,3,4, \\ \frac{1}{d_{e}} f_{e}\left(u_{j}\right)+2 u_{j}-1, & j=1,2,5 .\end{cases}
$$

3.

$$
\frac{2}{d_{e}} f_{e}^{\prime}(u)+3>0 \text { for } \begin{cases}0 \leq u \leq u_{j}, & j=0,1,2 \\ u_{j} \leq u \leq 1, & j=3,4,5\end{cases}
$$

Then $\Phi$ has

1. a countable infinity of periodic orbits of arbitrarily high period,

2. an uncountable infinity of nonperiodic orbit, and

3. a dense orbit.

Proof. The proof uses arguments similar to that of Lemma 9; that is, we need to find 12 points that satisfy the Conley-Moser conditions in this setup. The following table summarizes the mapping of the points and the conditions that the points $u_{j}, u_{j}^{*}$ must satisfy:

\begin{tabular}{|l|l|l|}
\hline$\Phi\left(U_{0}\right)=U_{0}^{*}$ & $\frac{2}{d_{e}} f_{e}\left(u_{0}\right)+3 u_{0}+\frac{1}{d_{o}} f_{o}\left(\frac{1}{d_{e}} f_{e}\left(u_{0}\right)+2 u_{0}\right)=1$ & $u_{0}^{*}=\frac{1}{d_{e}} f_{e}\left(u_{0}\right)+2 u_{0}$ \\
\hline$\Phi\left(U_{1}\right)=U_{1}^{*}$ & $\frac{2}{d_{e}} f_{e}\left(u_{1}\right)+3 u_{1}-2+\frac{1}{d_{o}} f_{o}\left(\frac{1}{d_{e}} f_{e}\left(u_{1}\right)+2 u_{1}-1\right)=0$ & $u_{1}^{*}=\frac{1}{d_{e}} f_{e}\left(u_{1}\right)+2 u_{1}-1$ \\
\hline$\Phi\left(U_{2}\right)=U_{2}^{*}$ & $\frac{2}{d_{e}} f_{e}\left(u_{2}\right)+3 u_{2}-2+\frac{1}{d_{o}} f_{o}\left(\frac{1}{d_{e}} f_{e}\left(u_{2}\right)+2 u_{2}-1\right)=1$ & $u_{2}^{*}=\frac{1}{d_{e}} f_{e}\left(u_{2}\right)+2 u_{2}-1$ \\
\hline$\Phi\left(U_{3}\right)=U_{3}^{*}$ & $\frac{2}{d_{e}} f_{e}\left(u_{3}\right)+3 u_{3}+\frac{1}{d_{o}} f_{o}\left(\frac{1}{d_{e}} f_{e}\left(u_{3}\right)+2 u_{3}\right)=0$ & $u_{3}^{*}=\frac{1}{d_{e}} f_{e}\left(u_{3}\right)+2 u_{3}$ \\
\hline$\Phi\left(U_{4}\right)=U_{4}^{*}$ & $\frac{2}{d_{e}} f_{e}\left(u_{4}\right)+3 u_{4}+\frac{1}{d_{o}} f_{o}\left(\frac{1}{d_{e}} f_{e}\left(u_{4}\right)+2 u_{4}\right)=1$ & $u_{4}^{*}=\frac{1}{d_{e}} f_{e}\left(u_{4}\right)+2 u_{4}$ \\
\hline$\Phi\left(U_{5}\right)=U_{5}^{*}$ & $\frac{2}{d_{e}} f_{e}\left(u_{5}\right)+3 u_{5}-2+\frac{1}{d_{o}} f_{o}\left(\frac{1}{d_{e}} f_{e}\left(u_{5}\right)+2 u_{5}-1\right)=0$ & $u_{5}^{*}=\frac{1}{d_{e}} f_{e}\left(u_{5}\right)+2 u_{5}-1$ \\
\hline
\end{tabular}

Copyright $\odot$ by SIAM. Unauthorized reproduction of this article is prohibited. 
In this table, we use a notation (see (5.13)) similar to the approximating case, where the hats in $u_{j}, u_{j}^{*}$ have been dropped. Each of the equations in the second column in this table can be written in the form $u_{j}=F\left(u_{j}^{*}\right)$, where $u_{j}^{*}$ is defined in the third column. For example, since $u_{0}^{*}=\frac{1}{d_{e}} f_{e}\left(u_{0}\right)+2 u_{0}$, the second column for $u_{0}$ gives $2 u_{0}^{*}-u_{0}+\frac{1}{d_{o}} f_{o}\left(u_{0}^{*}\right)=1$, or $u_{0}=\frac{1}{d_{o}} f_{o}\left(u_{0}^{*}\right)+2 u_{0}^{*}-1$.

Next, we want to find sufficient conditions that will guarantee the existence of the 12 points $u_{j}, u_{j}^{*}$. To this end, let us rewrite the equations for $u_{j}, u_{j}^{*}$ in the following way. Define

$$
\epsilon_{j}=\frac{f_{o}\left(u_{j}^{*}\right)}{d_{o}}, \quad j \in\{0,1,2,3,4,5\}
$$

where

$$
u_{j}^{*}= \begin{cases}\frac{1}{d_{e}} f_{e}\left(u_{j}\right)+2 u_{j}, & j=0,3,4, \\ \frac{1}{d_{e}} f_{e}\left(u_{j}\right)+2 u_{j}-1, & j=1,2,5 .\end{cases}
$$

Since $a_{o}<0$, we see that each perturbation $\epsilon_{j}$ is negative. For each $j$, observe that $\Phi\left(U_{j}\right)-$ $\hat{\Phi}\left(U_{j}\right)=E\left(U_{j}\right)=\left(\epsilon_{j}, 0\right)$.

Define the following family of functions (see (5.12)):

$$
H_{-l}(u)=\frac{2}{d_{e}} f_{e}(u)+3 u-l, \quad l \in \mathbb{R} .
$$

Then the equations in the second column can be written as

$$
H_{-\left(1-\epsilon_{0}\right)}\left(u_{0}\right)=H_{-\left(2-\epsilon_{1}\right)}\left(u_{1}\right)=H_{-\left(3-\epsilon_{2}\right)}\left(u_{2}\right)=H_{\epsilon_{3}}\left(u_{3}\right)=H_{-\left(1-\epsilon_{4}\right)}\left(u_{4}\right)=H_{-\left(2-\epsilon_{5}\right)}\left(u_{5}\right)=0 .
$$

Thus, if we assume that $-1<\epsilon_{j}<0$ for each $j$, then we have the following ordering of the family of functions $H_{-l}$, where $l \in \mathbb{R}$ :

$$
H_{0}>H_{\epsilon_{3}}>H_{-1}>H_{-\left(1+m_{0,4}\right)}>H_{-\left(1+M_{0,4}\right)}>H_{-\left(2+m_{1,5}\right)}>H_{-\left(2+M_{1,5}\right)}>H_{-3}>H_{-\left(3-\epsilon_{2}\right)},
$$

where

$$
\begin{array}{ll}
m_{0,4}=\min \left\{-\epsilon_{0},-\epsilon_{4}\right\}, & M_{0,4}=\max \left\{-\epsilon_{0},-\epsilon_{4}\right\}, \\
m_{1,5}=\min \left\{-\epsilon_{1},-\epsilon_{5}\right\}, & M_{1,5}=\max \left\{-\epsilon_{1},-\epsilon_{5}\right\} .
\end{array}
$$

Because of this ordering of the translates, the numbers $u_{j}$ exist if each of the top and bottom translates, $H_{0}$ and $H_{-\left(3-\epsilon_{2}\right)}$, has three distinct real zeroes. The maximum real zero of $H_{0}$ is the number $u_{3}$ while the minimum real zero of $H_{-\left(3-\epsilon_{2}\right)}$ is $u_{2}$. Sufficient conditions for the existence of three distinct real roots of $H_{0}$ and $H_{-\left(3-\epsilon_{2}\right)}$ are $H_{0}\left(S_{+}\right)<0<H_{-\left(3-\epsilon_{2}\right)}\left(S_{-}\right)$, which we can also write as

$$
H_{0}\left(S_{+}\right)<0<H_{0}\left(S_{-}\right)-3+\epsilon_{2},
$$

Copyright (c) by SIAM. Unauthorized reproduction of this article is prohibited. 
where $\left(S_{-}, S_{+}\right)$is the spinodal interval of $H_{-l}$, that is,

$$
S_{ \pm}=\frac{a_{e}+1 \pm \sqrt{a_{e}^{2}-a_{e}+1-\frac{9 d_{e}}{2 \tau_{1}^{2}}}}{3} .
$$

Finally, a sufficient condition for $\epsilon_{j}>-1$ is that $f_{o}\left(s_{+}\right)>-d_{o}$, where $s_{+}$is the right endpoint of the spinodal interval of $f_{o}$, that is, $\min _{0 \leq u \leq 1} f_{o}(u)=f_{o}\left(s_{+}\right)$:

$$
s_{+}=\frac{a_{o}+1+\sqrt{a_{o}^{2}-a_{o}+1}}{3} ;
$$

in particular, $\epsilon_{2}>\frac{f_{o}\left(s_{+}\right)}{d_{o}}$. Choose $\delta \in(-1,0)$ such that $\epsilon_{2}>\delta>\frac{f_{o}\left(s_{+}\right)}{d_{o}}$. Hence, if $H_{0}\left(S_{-}\right)-$ $3+\delta>0$, then $H_{0}\left(S_{-}\right)-3+\epsilon_{2}>0$.

The next lemma collects all the requirements that $a, d$ must satisfy in order to ensure that $u_{j}, u_{j}^{*}$ exist; this is a result similar to Lemma 10. The first requirement is needed to guarantee that the translates are ordered as in (5.26) while the last two requirements guarantee that the top and bottom translates $H_{0}$ and $H_{-\left(3-\epsilon_{2}\right)}$ have three distinct real zeroes. Finally, because the translates $H_{-k}$ for $k=0,1,2,3$ are squeezed in between $H_{0}$ and $H_{-\left(3-\epsilon_{2}\right)}$, the proof of Lemma 10 will follow from the proof of the following lemma.

Lemma 12. Consider the connection $(0,0)$ to $\left(\tau_{1}, \tau_{2}\right)$ for a fixed $(d, a)$ in a bistable region B. Assume that

1. $f_{o}\left(s_{+}\right)+d_{o}>0$,

2. $a_{e}^{2}-a_{e}+1-\frac{9 d_{e}}{2 \tau_{1}^{2}}>0$, and

3. $H_{0}\left(S_{+}\right)<0<H_{0}\left(S_{-}\right)-3+\delta$,

where $S_{+}, S-, s_{-}$are defined by (5.27), (5.28), and $\delta \in(-1,0)$ satisfies $\delta d_{o}-f_{o}\left(s_{+}\right)>0$. Then $\Phi$ possesses the shift on sequences on two symbols as a subsystem.

It is not easy to untangle the three conditions in Lemma 12 to get a pinning interval of the form similar to Lemma 10. To see that the above three conditions are viable assumptions, we apply a continuity argument on the parameters. Indeed, as $d \rightarrow 0-$, we have that $f_{o} \rightarrow$ $0, d_{o} \rightarrow a / 2$ so that the first requirement, asymptotically, is $a>0$; as $d \rightarrow 0-$, we have that $a_{e} \rightarrow a, d_{e} \rightarrow 0$ so that the second requirement is $1>0$; finally, as $d \rightarrow 0-$, we have that $S_{ \pm} \rightarrow T_{ \pm}$, where $\left(T_{-}, T_{+}\right)$is the spinodal interval (4.9) of $f_{a}$ so that the third inequality is $f_{a}\left(T_{+}\right)<0<f_{a}\left(T_{-}\right)$(recall that $0<a<1$ ).

5.1.3. Minimum wave speed. The Fisher equation [13] is an example of a monostable scalar reaction-diffusion equation:

$$
\frac{\partial u}{\partial t}=d u_{x x}+u(1-u), \quad d>0 .
$$

The monostable scalar spatially discrete reaction-diffusion LDE is

$$
\dot{u}_{n}(t)=d\left(u_{n-1}-2 u_{n}+u_{n+1}\right)-u_{n}\left(u_{n}-1\right), \quad d>0, n \in \mathbb{Z} .
$$

Let $f(u)=u(u-1)$, the Fisher nonlinearity. In both the PDE and the LDE, we have that $f(0)=f(1)=0$ for $0<u<1$ and $f^{\prime}(1)<0<f^{\prime}(0)$; that is, the equilibrium point 0 is 
unstable while 1 is stable. The existence of a monotone traveling wavefront solution connecting 0 to 1 depends on the wave speed $c$ - there is a number $c_{*}>0$ such that a monotone traveling wavefront solution $u(x, t)=U(x-c t$ ) (where $x \in \mathbb{R}$ for the PDE case while $x \in \mathbb{Z}$ for the LDE case) exists if and only if $c \geq c_{*}$. In other words, $c_{*}$ is the smallest value of $c$ for which there exists a monotone traveling wavefront and it is obtained by studying the linear stability of the equilibria of the system that is obtained by applying the traveling wave ansatz.

For the PDE case, a necessary condition for a monotone traveling wavefront solution to exist is

$$
c \geq 2 \sqrt{f^{\prime}(0)}
$$

while a sufficient condition for a monotone traveling wave front to exist is

$$
c \geq 2 \sqrt{\beta}, \quad \beta=\sup \left\{\frac{f(u)}{u}: 0<u<1\right\}
$$

that is, $2 \sqrt{f^{\prime}(0)} \leq c_{*} \leq 2 \sqrt{\beta}$. These are well-known results; for example, see [11]. For the Fisher nonlinearity, we have that $c_{*}=2$ because $f^{\prime}(0)=1=\beta$. The speed $c_{*}$ is sometimes referred to as the linear minimum wave speed. In general, however, $\beta \neq f^{\prime}(0)$ and for some choices of $f, c_{*}>2$ (see [3]).

For the LDE case, the first results were obtained by Harris, Hudson, and Zinner [17] for the scalar case and by Hudson and Zinner [19] for the periodic case. In the scalar LDE case [17], if $f$, like the Fisher nonlinearity, satisfies the extra assumption $f^{\prime}(0) x \geq f(x)$ for $x>0$, then there is a traveling wavefront solution if and only if $d \leq \sup _{\lambda>0} \frac{\lambda c-f^{\prime}(0)}{4 \sinh ^{2}(\lambda / 2)}$, equivalently,

$$
c \geq \inf _{\lambda>0} \frac{4 d \sinh ^{2}(\lambda / 2)+f^{\prime}(0)}{\lambda} .
$$

In the periodic LDE case, Hudson and Zinner [19] obtained a sufficient condition for the existence of traveling wavefronts. A more recent study was conducted by Guo and Hamel [14], where they were able to obtain sufficient and necessary conditions for the existence of traveling wavefronts; however, their results are not directly applicable to the antidiffusion lattice Nagumo problem because [14] studied a slightly different system of periodic LDEs, where a periodic LDE that is in divergence form:

$$
\dot{u}_{n}(t)=d_{n+1} u_{n+1}(t)+d_{n} u_{n-1}(t)-\left(d_{n+1}+d_{n}\right) u_{n}(t)+f\left(u_{n}\right), \quad t \in \mathbb{Z} .
$$

They obtained that a sufficient and necessary condition for the existence of traveling wavefronts is that $c \geq c_{*}$, where $c_{*}=\min _{\lambda>0} \frac{M(\lambda)}{\lambda}$, where $M(\lambda)$ is the largest real eigenvalue of a certain matrix.

Let us now proceed to study the minimum wave speed for the antidiffusion lattice Nagumo problem in a region $M$ where monostable dynamics occurs for the connection from $(0,0)$ to $\left(\tau_{1}, \tau_{2}\right)$. Note that by our previous computations, $M$ is a subset of

$$
\{(d, a): a+4 d<0,0<a<1\} .
$$

Copyright $\odot$ by SIAM. Unauthorized reproduction of this article is prohibited. 
Negative diffusion. Consider the matrix $A(\lambda)$ defined for $\lambda>0$ by

$$
A(\lambda)=\left(\begin{array}{cc}
-\left(2 d_{e}+f_{e}^{\prime}(0)\right) & 2 d_{e} \cosh \lambda \\
2 d_{o} \cosh \lambda & -\left(2 d_{o}+f_{o}^{\prime}(0)\right)
\end{array}\right) .
$$

Note that $A(\lambda=0)$ gives the linearization matrix at the point $(0,0)$. Let $M(\lambda)$ denote the largest positive eigenvalue of $A(\lambda)$. The eigenvalues of $A(\lambda)$ are given by $\frac{T \pm \sqrt{T^{2}-4 D(\lambda)}}{2}$, where $T$ is the trace given by $T=-2\left(d_{e}+d_{o}\right)-\left(f_{e}^{\prime}(0)+f_{o}^{\prime}(0)\right)$ and $D(\lambda)$ is the determinant given by $D(\lambda)=\left(2 d_{e}+f_{e}^{\prime}(0)\right)\left(2 d_{o}+f_{o}^{\prime}(0)\right)-4 d^{2} \cosh ^{2} \lambda$. The discriminant is always nonnegative:

$$
T^{2}-4 D(\lambda)=\left(\left(2 d_{e}+f_{e}^{\prime}(0)\right)-\left(2 d_{o}+f_{o}^{\prime}(0)\right)\right)^{2}+16 d^{2} \cosh ^{2} \lambda=16 d^{2} \cosh ^{2} \lambda \geq 0
$$

by using (2.11), and hence the eigenvalues of $A(\lambda)$ are both real.

The trace of $A(\lambda)$ is equal to

$$
\begin{aligned}
T & =-\left(2 d_{e}+f_{e}^{\prime}(0)+2 d_{o}+f_{o}^{\prime}(0)\right) \\
& =-2 d_{e}-\left(a-2\left(d_{e}-d\right)\right)-2 d_{o}-\left(a-2\left(d_{o}-d\right)\right) \\
& =-2(a+2 d) .
\end{aligned}
$$

Denote the larger eigenvalue of $A(\lambda)$ by $M(\lambda)$. Then, noting that the minimum of $\cosh \lambda$ is 1 , we have

$$
M(\lambda)=\left(T+\sqrt{T^{2}-4 D(\lambda)}\right) / 2=-(a+2 d)-2 d \cosh \lambda \geq-(a+4 d)>0 .
$$

In particular, $M(0)=-(a+4 d)>0$. Since $M^{\prime}(\lambda)=-2 d \sinh \lambda$, we have $M^{\prime}(0)=0$. Hence, the minimum of $M(\lambda) / \lambda$ over all $\lambda>0$ is achieved and is positive. We define $c_{*}$ :

$$
c_{*}=\min _{\lambda>0} \frac{M(\lambda)}{\lambda}
$$

This value $c_{*}$ is the so-called minimum wave speed for the traveling wavefront solution that connects $(0,0)$ to $\left(\tau_{1}, \tau_{2}\right)$. Then, using an expansion of $\cosh \lambda$, we have

$$
\begin{aligned}
\frac{M(\lambda)}{\lambda} & =\frac{-a-2 d(1+\cosh \lambda)}{\lambda} \\
& =\frac{-a}{\lambda}-2 d\left(\frac{2}{\lambda}+\frac{\lambda}{2 !}+\frac{\lambda^{3}}{4 !}+\frac{\lambda^{5}}{6 !}+\cdots\right) \\
& >\frac{-a-4 d}{\lambda}-d \lambda \\
& =\frac{-a-4 d-d \lambda^{2}}{\lambda}:=G(\lambda)
\end{aligned}
$$

for $\lambda>0$. We see that $G^{\prime}(\lambda)=0<G^{\prime \prime}(\lambda)$ when $\lambda=\sqrt{\frac{a+4 d}{d}}$; that is, $G$ is minimized at this $\lambda$. Thus, we have computed a (positive) lower bound for $c^{*}$ :

$$
c^{*} \geq G\left(\sqrt{\frac{a+4 d}{d}}\right)=2 \sqrt{d(a+4 d)} .
$$

Copyright $($ ) by SIAM. Unauthorized reproduction of this article is prohibited. 


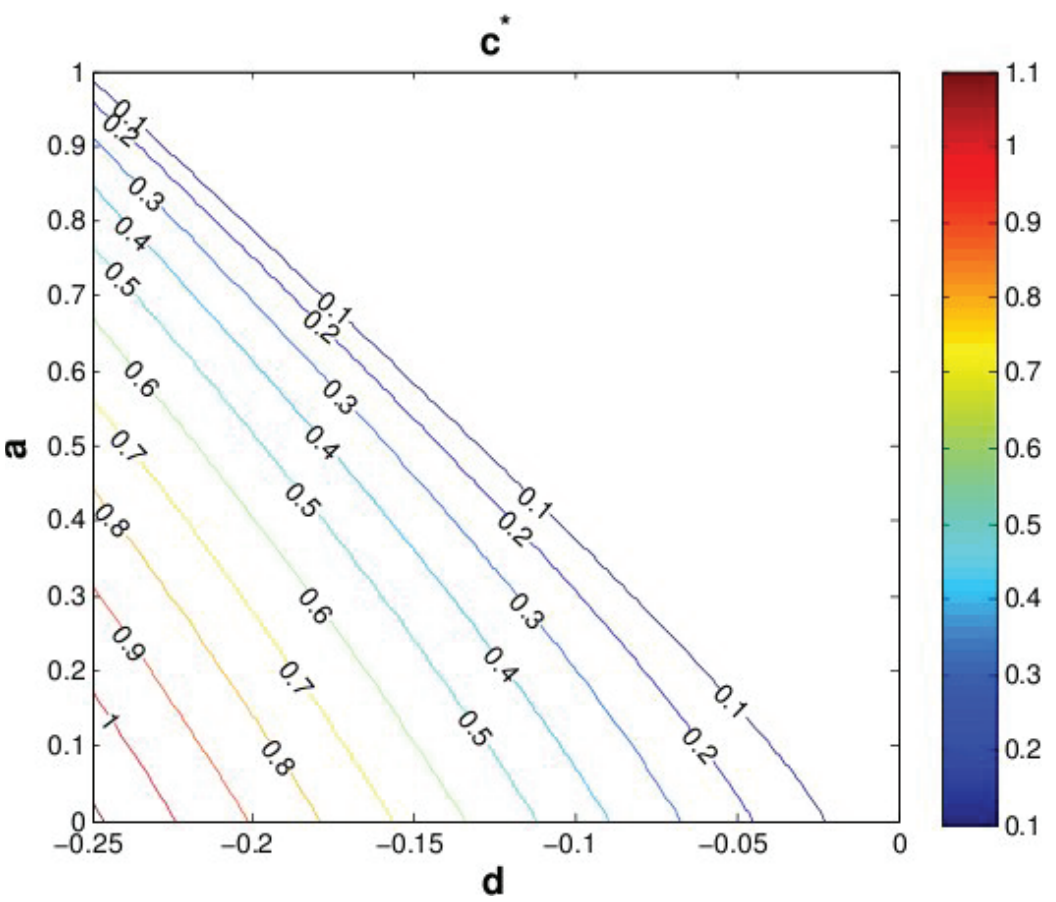

Figure 12. This is a contour plot of $c_{*}$ for $-1 / 4<d<0$ and $0<a<1$.

Compare this lower bound for $c_{*}$ with the expression for the monostable scalar PDE case (5.29). In Figure 12 we show the computed values of the minimum wave speed $c_{*}$ for the antidiffusion lattice Nagumo problem for $a+4 d<0$ and $0<a<1$.

Finally, using a hyperbolic identity, we can further rewrite our result (compare with 5.30) in the following way:

$$
c_{*}=\min _{\lambda>0} \frac{M(\lambda)}{\lambda}=\min _{\lambda>0} \frac{-a-2 d(1+\cosh \lambda)}{\lambda}=\min _{\lambda>0} \frac{-f_{a}^{\prime}(0)-4 d \cosh ^{2}(\lambda / 2)}{\lambda} .
$$

5.2. Case study: $\left(\tau_{2}, \tau_{1}\right)$ to $\left(\tau_{1}, \tau_{2}\right)$ connection. In this case, we want to know whether there exists a traveling wavefront solution to $(2.2)$ that connects $\left(x_{-}, y_{-}\right)=\left(\tau_{2}, \tau_{1}\right)$ and $\left(x_{+}, y_{+}\right)=\left(\tau_{1}, \tau_{2}\right)$. Here, $\alpha=-\beta=\tau_{1}-\tau_{2}>0$, and the diffusion-coupling parameters are $d_{e}=-d=d_{o}$. This case study is interesting because the boundary conditions are either both stable or both unstable (by Lemma 4), and hence monostable dynamics cannot occur. Furthermore, unlike the first case study, the intermediate point is not unique. In fact, in the regions where there are nine equilibria, there are seven intermediate points; where there are seven equilibria, there are five intermediate points; and where there are five equilibria, there are three intermediate points.

For example, consider the region

$$
R=\{(d, a): a+4 d<0,1-a+4 d<0, a(a-1)+4 d<0\} .
$$

This is the region in Figure 1 where there are only five distinct real zeroes to the ninth-degree polynomial $g$. The set $R$ is quadrant $I I I_{\text {in }}$ in the proof of Lemma 2. In $R$, for any value 
of $a$, the points $\left(0^{ \pm}, 0^{\mp}\right),\left(1^{ \pm}, 1^{\mp}\right),\left(a^{ \pm}, a^{\mp}\right)$ do not exist and the points $(0,0),(a, a),(1,1)$ are unstable.

Thus, all three intermediate points between $\left(\tau_{2}, \tau_{1}\right)$ and $\left(\tau_{1}, \tau_{2}\right)$ are unstable. If $f_{a}^{\prime}\left(\tau_{1}\right)+$ $2 d>0$ in $R$, then the boundary conditions are stable so that the antidiffusion lattice Nagumo system has bistable dynamics. Since $\tau_{1}>1$, we have that $2 d+f_{a}^{\prime}\left(\tau_{1}\right)>0$ if $1-a+2 d>0$. Therefore, the set of parameter values in the set

$$
B=\{a+4 d<0,1-a+2 d>0>1-a+4 d, a(a-1)+4 d<0\}
$$

will give rise to bistable dynamics to the antidiffusion lattice Nagumo system.

For completeness (see section 5.3), let us look at the case where $E$ has nine equilibria and determine which of the intermediate points from $\left(\tau_{2}, \tau_{1}\right)$ to $\left(\tau_{1}, \tau_{2}\right)$ will give rise to bistable dynamics, if any. Consider the set of parameter values in the region

$$
T=\{(d, a): a+4 d>0,1-a+4 d>0\}
$$

this is quadrant $I$ in the proof of Lemma 2, where the equilibria $\left(0^{ \pm}, 0^{\mp}\right)$ and $\left(1^{ \pm}, 1^{\mp}\right)$ exist. Since $1-a+4 d>0$ implies that $1-a+2 d>0$, we have $2 d+f_{a}^{\prime}\left(\tau_{1}\right)>0$; that is, the boundary conditions $\left(\tau_{1}, \tau_{2}\right)$ and $\left(\tau_{2}, \tau_{1}\right)$ are both stable. Bistable dynamics will not occur in this case because there are more than one stable intermediate points, namely $(0,0)$ and $(1,1)$. In fact, if $2 d+f_{a}^{\prime}\left(0^{+}\right)<0$ and $2 d+f_{a}^{\prime}\left(1^{+}\right)<0$, we see that in the chain from $\left(x_{-}, y_{-}\right)$to $\left(x_{+}, y_{+}\right)$, there are four stable equilibria, denoted in bold:

$$
\left(\boldsymbol{\tau}_{2}, \boldsymbol{\tau}_{\mathbf{1}}\right) \rightarrow\left(0^{-}, 0^{+}\right) \rightarrow(\mathbf{0}, \mathbf{0}) \rightarrow\left(0^{+}, 0^{-}\right) \rightarrow(a, a) \rightarrow\left(1^{-}, 1^{+}\right) \rightarrow(\mathbf{1}, \mathbf{1}) \rightarrow\left(1^{+}, 1^{-}\right) \rightarrow\left(\boldsymbol{\tau}_{1}, \boldsymbol{\tau}_{\mathbf{2}}\right) .
$$

5.3. Co-existence. In this final section of examples, we show that there are regions in the parameter space where bistable and monostable dynamics in the antidiffusion lattice Nagumo system co-exist. Define the parameter region

$$
T=\{(d, a): a+4 d>0,1-a+4 d>0\}
$$

this is quadrant $I$ in the proof of Lemma 2, where $0<a<1$. To show that bistable and monostable dynamics can both occur in $T$, we look at the stability of each of the nine equilibria.

Since $1-a+2 d>0$ in $T$, the equilibria $\left(\tau_{1}, \tau_{2}\right)$ and $\left(\tau_{2}, \tau_{1}\right)$ are stable. Also, for $(d, a)$ in $T$, both $(0,0)$ and $(1,1)$ are stable because $a+4 d>0$ and $1-a+4 d>0$, respectively; however, $(a, a)$ is unstable because $a(a-1)+4 d<0$. The equilibria $\left(0^{ \pm}, 0^{\mp}\right)$ are unstable if $2 d+f_{a}^{\prime}\left(0^{+}\right)<0$ and the equilibria $\left(1^{ \pm}, 1^{\mp}\right)$ are unstable if $2 d+f_{a}^{\prime}\left(1^{+}\right)<0$.

By ellipticity, we must require that $\alpha \beta<0$. Hence, for example, even if $(0,0)$ and $(1,1)$ are both stable, the theory cannot be applied with these as boundary conditions because $\alpha=\beta=1$. The following table summarizes the connections that exist between any two equilibria in $\hat{E}$ that satisfy $\alpha \beta<0$ and whether there is bistable or monostable dynamics. In

Copyright $\odot$ by SIAM. Unauthorized reproduction of this article is prohibited. 
the table, $\gamma \in\{0,1\}$ and $\gamma^{ \pm} \in\left\{0^{ \pm}, 1^{ \pm}\right\}$.

\begin{tabular}{|l|l|l|l|}
\hline$\left(x_{-}, y_{-}\right)$ & $\left(x_{+}, y_{+}\right)$ & Intermediate points & Monostable or bistable \\
\hline$\left(\tau_{2}, \tau_{1}\right)$ & $\left(0^{-}, 0^{+}\right)$ & none & monostable dynamics \\
& $(0,0)$ & $\left(0^{-}, 0^{+}\right)$ & bistable dynamics if $2 d+f_{a}^{\prime}\left(0^{+}\right)<0$ \\
& $(a, a)$ & none & monostable dynamics \\
& $\left(1^{-}, 1^{+}\right)$ & none & monostable dynamics \\
& $(1,1)$ & $\left(1^{-}, 1^{+}\right)$ & bistable dynamics if $2 d+f_{a}^{\prime}\left(1^{+}\right)<0$ \\
& $\left(\tau_{1}, \tau_{2}\right)$ & other 7 equilibria & see section 5.2 \\
\hline$\left(\gamma^{\mp}, \gamma^{ \pm}\right)$ & $\left(\tau_{2}, \tau_{1}\right)$ & none & monostable dynamics \\
& $(\gamma, \gamma)$ & none & monostable dynamics \\
\hline$(\gamma, \gamma)$ & $\left(\tau_{2}, \tau_{1}\right)$ & $\left(\gamma^{-}, \gamma^{+}\right)$ & bistable dynamics if $2 d+f_{a}^{\prime}\left(\gamma^{+}\right)<0$ \\
& $\left(\tau_{1}, \tau_{2}\right)$ & $\left(\gamma^{+}, \gamma^{-}\right)$ & bistable dynamics if $2 d+f_{a}^{\prime}\left(\gamma^{+}\right)<0$ \\
& $\left(\gamma^{-}, \gamma^{+}\right)$ & none & monostable dynamics \\
& $\left(\gamma^{+}, \gamma^{-}\right)$ & none & monostable dynamics \\
\hline$(a, a)$ & $\left(\tau_{2}, \tau_{1}\right)$ & none & monostable dynamics \\
& $\left(\tau_{1}, \tau_{2}\right)$ & none & monostable dynamics \\
\hline$\left(\tau_{1}, \tau_{2}\right)$ & $\left(\tau_{2}, \tau_{1}\right)$ & other 7 equilibria & see section 5.2 \\
& $(0,0)$ & $\left(0^{+}, 0^{-}\right)$ & bistable dynamics if $2 d+f_{a}^{\prime}\left(0^{+}\right)<0$ \\
& $\left(0^{+}, 0^{-}\right)$ & none & monostable dynamics \\
& $(a, a)$ & none & monostable dynamics \\
& $(1,1)$ & $\left(1^{+}, 1^{-}\right)$ & bistable dynamics if $2 d+f_{a}^{\prime}\left(1^{+}\right)<0$ \\
& $\left(1^{+}, 1^{-}\right)$ & none & monostable dynamics \\
\hline
\end{tabular}

To determine whether there is monostable or bistable dynamics from $\left(x_{-}, y_{-}\right)$to $\left(x_{+}, y_{+}\right)$, we determine the stability of $\left(x_{ \pm}, y_{ \pm}\right)$and the intermediate point, if such intermediate points exist. From the table, we observe, for example, assuming $2 d+f_{a}^{\prime}\left(0^{+}\right)<0$, that there is monostable dynamics from $\left(\tau_{2}, \tau_{1}\right)$ to $\left(0^{-}, 0^{+}\right)$and from $\left(0^{-}, 0^{+}\right)$to $(0,0)$ while there is bistable dynamics from $\left(\tau_{2}, \tau_{1}\right)$ to $(0,0)$; that is, like the positive diffusion problem, bistable dynamics may be viewed as a concatenation of two monostable dynamics that share an equilibrium.

6. Conclusions. In this paper, we considered a specific lattice model of phase transitions where the diffusion term has a negative coefficient and the reaction term is the smooth cubic nonlinearity. We have discovered a rich dynamical phenomenon that includes both bistable and monostable behavior. We applied recent results to establish the existence, uniqueness, and stability of traveling wavefront solutions in discrete periodic media and found a plethora of connecting orbits. Avenues for future work include more complicated models of this type (we are considering here an overdamped limit) and analogous problems in higher space dimensions.

Acknowledgments. We are grateful to Anna Vainchtein and the referees for helpful remarks and suggestions that improved the paper.

\section{REFERENCES}

[1] D. Aronson and H. Weinberger, Nonlinear diffusion in population genetics, combustion, and nerve pulse propagation, in Partial Differential Equations and Related Topics, Lecture Notes in Math. 446, Springer-Verlag, Berlin, 1975, pp. 5-49.

Copyright ( $\odot$ by SIAM. Unauthorized reproduction of this article is prohibited. 
[2] J. Bell AND C. Cosner, Threshold behavior and propagation for nonlinear differential-difference systems motivated by modeling myelinated axons, Quart. Appl. Math., 42 (1984), pp. 1-14.

[3] R.D. Benguria And M.C. Depassier, Validity of the linear speed selection mechanism for fronts of the nonlinear diffusion equation, Phys. Rev. Lett., 73 (1994), pp. 2272-2274.

[4] J. CAhn, S.N. Chow, And E.S. VAN Vleck, Spatially discrete nonlinear diffusion equations, Rocky Mountain J. Math., 25 (1995), pp. 87-118.

[5] J.W. Cahn, J. Mallet-Paret, and E.S. Van Vleck, Traveling wave solutions for systems of ODEs on a two-dimensional spatial lattice, SIAM J. Appl. Math., 59 (1998), pp. 455-493.

[6] X. Chen, S.-C. Fu, And J.-S. Guo, Uniqueness and asymptotics of traveling waves of monostable dynamics on lattices, SIAM J. Math. Anal., 38 (2006), pp. 233-258.

[7] X. Chen, J.-S. Guo, And C.-C. Wu, Traveling waves in discrete periodic media for bistable dynamics, Arch. Ration. Mech. Anal., 189 (2008), pp. 189-236.

[8] S.-N. Chow, J. Mallet-Paret, and E.S. VAn Vleck, Dynamics of lattice differential equations, Internat. J. Bifur. Chaos Appl. Sci. Engrg., 6 (1996), pp. 1605-1621.

[9] C. Elmer, Finding stationary fronts for a discrete Nagumo and wave equation; construction, Phys. D, 218 (2006), pp. 11-23.

[10] T. ERneux and G. Nichols, Propagating waves in discrete bistable reaction-diffusion systems, Phys. D, 67 (1993), pp. 237-244.

[11] P.C. Fife, Mathematical Aspects of Reacting and Diffusing Systems, Springer-Verlag, New York, Berlin, 1979.

[12] P.C. FifE AND J.B. MCLEOD, The approach of solutions of nonlinear diffusion equations to traveling front solutions, Arch. Ration. Mech. Anal., 65 (1977), pp. 335-361.

[13] R. Fisher, The wave advance of advantageous genes, Ann. Eugenics, 7 (1937), pp. 355-369.

[14] J.-S. Guo AND F. HAMEL, Front propagation for discrete periodic monostable equations, Math. Ann., 335 (2006), pp. 489-525.

[15] J.-S. GUO AND C.-C. WU, Uniqueness and stability of traveling waves for periodic monostable lattice dynamical system, J. Differential Equations, 246 (2009), pp. 3818-3833.

[16] D. Hankerson AND B. Zinner, Wavefronts for a cooperative tridiagonal system of differential equations, J. Dynam. Differential Equations, 5 (1993), pp. 359-373.

[17] G. Harris, W. Hudson, And B. Zinner, Traveling wavefronts for the discrete Fisher's equation, J. Differential Equations, 105 (1993), pp. 46-62.

[18] J. Harterich, B. Sandstede, And A. Scheel, Exponential dichotomies for linear non-autonomous functional differential equations of mixed type, Indiana Univ. Math. J., 51 (2002), pp. 1081-1109.

[19] W. Hudson AND B. Zinner, Existence of traveling waves for a generalized discrete Fisher's equation, Comm. Appl. Nonlinear Anal., 1 (1994), pp. 23-46.

[20] H.J. Hupkes And S.M. Verduyn-Lunel, Center manifold theory for functional differential equations of mixed type, J. Dynam. Differential Equations, 19 (2007), pp. 497-560.

[21] J.P. KeEner, Propagation and its failure in coupled systems of discrete excitable cells, SIAM J. Appl. Math., 47 (1987), pp. 556-572.

[22] J. Mallet-Paret, The Fredholm alternative for functional differential equations of mixed type, J. Dynam. Differential Equations, 11 (1999), pp. 1-48.

[23] J. MALLET-PARET The global structure of traveling waves in spatially discrete dynamical systems, J. Dynam. Differential Equations, 11 (1999), pp. 49-127.

[24] J. Mallet-PARet, Traveling waves in spatially discrete dynamical systems of diffusive type, in Dynamical Systems, Lecture Notes in Math. 1822, Springer-Verlag, Berlin, 2003, pp. 231-298.

[25] A. Vainchtein And E. VAn Vleck, Nucleation and propagation of phase mixtures in a bistable chain, Phys. Rev. B, 79 (2009), 144123.

[26] S. Wiggins, Introduction to Applied Nonlinear Dynamical Systems and Chaos, 2nd ed., Springer-Verlag, New York, 2000.

[27] B. ZINner, Stability of traveling wavefronts for the discrete Nagumo equation, SIAM J. Math. Anal., 22 (1991), pp. 1016-1020.

[28] B. Zinner, Existence of traveling wavefront solutions for the discrete Nagumo equation, J. Differential Equations, 96 (1992), pp. 1-27.

Copyright ( $\odot$ by SIAM. Unauthorized reproduction of this article is prohibited. 